\title{
THE
}

$11-2010$

\section{The Newsvendor Problem: Review and Directions for Future Research}

Yan Qin

Ruoxuan Wang

Asoo J. Vakharia

Yuwen Chen

University of Rhode Island, yuwen@uri.edu

Michelle MH Seref

Follow this and additional works at: https://digitalcommons.uri.edu/cba_facpubs

Terms of Use

All rights reserved under copyright.

\section{Citation/Publisher Attribution}

Yan Qin, Ruoxuan Wang, Asoo J. Vakharia, Yuwen Chen, and Michelle M. Seref. "The Newsvendor Problem: Review and Directions for Future Research" European Journal of Operational Research 213.2 (2011): 361-374. Available online at http://dx.doi.org/10.1016/j.ejor.2010.11.024.

This Article is brought to you for free and open access by the College of Business at DigitalCommons@URI. It has been accepted for inclusion in College of Business Faculty Publications by an authorized administrator of DigitalCommons@URI.For more information, please contact digitalcommons-group@uri.edu. 


\title{
The Newsvendor Problem: Review and \\ Directions for Future Research
}

\author{
Yan Qin ${ }^{1}$ \\ yan.qin@warrington.ufl.edu \\ Ruoxuan Wang ${ }^{1}$ \\ rxwang@ufl.edu \\ Asoo J. Vakharia ${ }^{1}$ \\ asoov@ufl.edu \\ Yuwen Chen ${ }^{2}$ \\ yuwen@mail.uri.edu \\ Michelle MH Seref ${ }^{3}$ \\ mmhseref@vt.edu
}

April 2009

revised: February 2010; June 2010; November 2010

${ }^{1}$ Department of Information Systems \& Operations Management, Warrington College of Business Administration, University of Florida, Gainesville, FL 32611.

${ }^{2}$ University of Rhode Island, Kingston, RI 02881.

${ }^{3}$ Virginia Polytechnic Institute and State University, Blacksburg, VA 24061-0002 


\title{
The Newsvendor Problem: Review and Directions for Future Research
}

\begin{abstract}
In this paper, we review the contributions to date for analyzing the newsvendor problem. Our focus is on examining the specific extensions for analyzing this problem in the context of modeling customer demand, supplier costs, and the buyer risk profile. More specifically, we analyze the impact of market price, marketing effort, and stocking quantity on customer demand; how supplier prices can serve as a coordination mechanism in a supply chain setting; integrating alternative supplier pricing policies within the newsvendor framework; and how the buyer's risk profile moderates the newsvendor order quantity decision. For each of these areas, we summarize the current literature and develop extensions. Finally, we also propose directions for future research.
\end{abstract}

\section{Introduction}

The newsvendor problem is one of the classical problems in the literature on inventory management ([7] [43]). Key insights stemming from an analysis of this problem have wide ranging implications for managing inventory decisions for organizations in, for example, the hospitality, airline, and fashion goods industries. A generic contemporary setting in which this problem could be framed is as follows. Consider a three-node supply chain consisting of a supplier, a buyer, and customers. At the beginning of a single period, the buyer is interested in determining an "optimal" stocking policy $(Q)$ to satisfy total customer demand for a single product. This customer demand is assumed to be stochastic and characterized by a random variable $x$ with the probability density function $f(x)$ and cumulative distribution function $F(x)$. The quantity $Q$ is purchased by the buyer from the supplier for a fixed price per unit $v$. The supplier is assumed to operate with no capacity restrictions and zero lead time of supply and thus, an order placed by the buyer with the supplier at the beginning of a period is immediately filled. Sales of the product occur during (or at the end of) the single period and: (a) if $Q \geq x$, then $Q-x$ units which are left over at the end of the period are salvaged by the buyer for a per unit revenue of $g^{1}$; and (b) if $Q<x$, then $x-Q$ units which represent "lost" sales cost the buyer $B$ per unit. Assuming a fixed market price of $p$, then the actual end of period profit for the buyer is:

$$
\Pi(Q, x)= \begin{cases}p x-v Q+g(Q-x) & \text { if } Q \geq x \\ p Q-v Q-B(x-Q) & \text { if } Q<x\end{cases}
$$

\footnotetext{
${ }^{1}$ For obvious reasons, it is generally assumed that $g<v$.
} 
Since the demand has not been realized at the beginning of the period, the buyer cannot observe the actual profit. Hence, the traditional approach to analyze the problem is based on assuming a risk neutral buyer who makes the optimal quantity decision at the beginning of the period by maximizing total expected profits. These profits are:

$$
\begin{aligned}
E[\Pi(Q)] & =\int_{0}^{Q}[p x-v Q+g(Q-x)] f(x) d x+\int_{Q}^{\infty}[p Q-v Q-B(x-Q)] f(x) d x \\
& =(p-g) \mu-(v-g) Q-(p-g+B) E S(Q)
\end{aligned}
$$

where $E[\cdot]$ is the expectation operator, $\mu$ is the mean demand, and $E S(Q)$ represents the expected units short assuming $Q$ units are stocked and can be determined as $\int_{Q}^{\infty}(x-Q) f(x) d x$. It is relatively easy to show that equation (2) is strictly concave in $Q$ (Silver, Pyke, and Peterson [43]) and hence, the first order conditions (FOCs) are necessary and sufficient to determine the value of $Q$ which maximizes equation (2). This optimal order quantity $\left(Q^{*}\right)$ is set such that:

$$
F\left(Q^{*}\right)=\frac{p-v+B}{p-g+B}
$$

and the corresponding profit for the buyer is:

$$
E\left[\Pi\left(Q^{*}\right)\right]=(p-g) \mu-(p-g+B) \int_{Q^{*}}^{\infty} x f(x) d x
$$

Prior researchers have sometimes adopted an "overage" $\left(c_{o}\right)$ and "underage" $\left(c_{u}\right)$ cost perspective in analyzing the model from an expected cost perspective. In this case, $F\left(Q^{*}\right)=\frac{c_{u}}{c_{o}+c u}$ where $c_{u}=p-v+B$ and $c_{o}=v-g$ and $E[\Pi(Q)]=(p-v) \mu-c_{o} \int_{0}^{Q}(Q-x) f(x) d x-c_{u} \int_{Q}^{\infty}(x-Q) f(x) d x$.

In the last decade, Khouja [30] has also reviewed the extensive contributions to the newsvendor problem. We extend this prior review by considering several specific extensions in our paper such as integrating marketing effort, stock dependent demand, and buyer risk profiles and how they all play a role in determining the optimal newsvendor order quantity. In addition, we also review 20 plus additional contributions since the review by Khouja [30]. We also refer the reader to a review of the newsvendor problem in supply chain settings with a primary focus on contractual mechanisms which is available in Cachon [15]. The motivation for our review, critique, and extensions stems from the fact that key parametric assumptions drive the relatively straightforward analysis for the single period newsvendor problem. In essence, our intent is on reviewing and examining how changes in the assumptions related to key parameters might moderate the current set of results. More specifically, this paper focuses on reviewing prior work and developing extensions related to the following:

1. Customer Demand: This is typically treated as an exogenous parameter in the prior analysis. However, depending upon the context, it could be argued that: 
- Customer demand $x$ and price $p$ are interrelated;

- Customer demand $x$ could be influenced by marketing effort $e$ expended by the buyer; and

- Customer demand $x$ could be influenced by the quantity stocked $Q$ by the buyer.

2. Supplier Pricing Policies: A fixed supplier price per unit $(v)$ quoted by the supplier is the standard assumption in the newsvendor setting. However, current practice (see, for example, Burke, Carrillo, and Vakharia [11]) indicates suppliers tend to quote quantity discount schemes to generate larger order quantities from the buyer. Thus, it would be interesting to examine how alternative supplier price discounting schemes might moderate the buyer's optimal quantity decision.

3. Buyer Risk Profile: The assumption underlying the basic structural result for the newsvendor model is that the buyer is risk neutral and thus, would choose to optimize expected profits. Given that sourcing practice often indicates that buyers are risk averse (Burke and Vakharia [13]) and/or "risk" takers, we focus on how such risk profiles would moderate the sourcing decision in a newsvendor setting.

The remainder of this paper is organized as follows. In the next section, we start by analyzing the impact of alternative customer demand related changes in a newsvendor setting; section 3 focuses on examining the impact of alternative supplier pricing schemes; and section 4 describes how the risk profile of the buyer impacts optimal decision making in this setting. Finally, implications and directions for future research are discussed in Section 5 .

\section{Customer Demand}

The analysis in this section focuses on how the optimal order quantity in a newsvendor setting would be impacted if demand was a function of: (a) market price; (b) marketing effort; and (c) stocking quantity.

\subsection{Demand and Market Price}

In a classic Newsvendor problem, selling price is considered as exogenous, over which the retailer has absolutely no control. This is true in a perfectly competitive market where buyers are mere pricetakers. In practice, however, retailers may adjust the current selling price in order to increase or reduce demand. Whitin [50], Mills [36] and Karlin and Carr [28] are three of the early publications 
that investigated the effects of different demand processes on the seller's pricing and ordering decisions.

Whitin [50] provided necessary optimality conditions for two models, an economic order quantity (EOQ) model and a style goods model. In contrast to the well-known EOQ approach which assumes an infinite planning horizon, a style goods model focuses on environments where there is a relatively short selling season with a well defined beginning and end. Although Whitin [50] only provided the closed-form solution for a style goods model with a rectangular demand distribution, he believed that structural solutions are possible for other cases provided the price-dependent demand distribution can be expressed as an explicit function of the expected demand.

Mills [36] assumed that the price-dependent demand $(x)$ is affected additively by a random term, $\epsilon$, independent of price, i.e. $x=\mu(p)+\epsilon$ where $\mu(p)$ is the mean demand as a function of market price $p$. In contrast to the deterministic demand model that optimizes at the price which equates riskless marginal revenue and marginal cost, the model under stochastic demand is optimized at the price which equates riskless marginal revenue to marginal cost plus an additional non-zero term. He concluded that the direction of change in the magnitude of the optimal price in the risky situation depends on the shape of the marginal cost curve and showed that this optimal price cannot be higher than it would be in the riskless case for a constant marginal cost. A contrary result was obtained by Karlin and Carr [28] for the case of a multiplicative model in which the stochastic demand $x=\mu(p) \epsilon$. The demand model employed in Mills [36] is referred to as the additive demand form and the one in Karlin and Carr [28] as the multiplicative demand form. In what follows, we will first derive some simple results for these two special demand-price relationships, and then review the analysis which leads to the results mentioned above.

In the additive demand case, assume that demand $x=\mu(p)+\epsilon$, where $\mu(p)=a-b p$. In this case, $a$ and $b$ are both positive constants, $p$ is the market price, and $\epsilon$ is a random variable with a probability density function $h(\cdot)$ and cumulative distribution function $H(\cdot)$ with a mean of zero. The expected profit function is now a bivariate function with price and order quantity as the decision variables and can be expressed in terms of overage and underage costs as:

$$
E[\Pi(p, Q)]=(p-v) \mu(p)-c_{o} \cdot E[Q-x]^{+}-c_{u} \cdot E[x-Q]^{+}
$$

where $E[Q-x]^{+}$is the expected number of units overstocked and $E[x-Q]^{+}$is the expected number of units understocked. By observing that $x=a+b p+\epsilon$, it is easy to show that (Petruzzi and Dada $[39])$ :

$$
E[Q-x]^{+} \quad=\int_{0}^{Q-a+b p} H(\epsilon) d \epsilon
$$




$$
E[x-Q]^{+}=\int_{Q-a+b p}^{+\infty}[1-H(\epsilon)] d \epsilon
$$

This expected profit can be interpreted as being equal to riskless profit minus expected cost. Next, let's consider the partial derivatives of the expected profit function with respect to $Q$ and $p$. Differentiating $E[\Pi(p, Q)]$ with respect to $Q$, we obtain

$$
\begin{gathered}
\frac{\partial E[\Pi(p, Q)]}{\partial Q}=c_{u}-\left(c_{o}+c_{u}\right) H(Q-a+b p) \\
\frac{\partial^{2} E[\Pi(p, Q)]}{\partial Q^{2}}=-\left(c_{o}+c_{u}\right) h(Q-a+b p)
\end{gathered}
$$

Clearly, $\frac{\partial^{2} E[\Pi(p, Q)]}{\partial Q^{2}} \leq 0$, which indicates that the expected profit function is concave in $Q$ for a given $p$. We can then solve for the optimal order quantity for a given $p$ by setting the FOC equal to zero. This results in:

$$
H\left(Q^{*}(p)-a+b p\right)=\frac{c_{u}}{c_{o}+c_{u}}
$$

where $Q^{*}(p)$ is the optimal order quantity ${ }^{2}$ for a given price $p$. After substituting $Q^{*}(p)=$ $H^{-1}\left[\frac{c_{u}}{c_{o}+c_{u}}\right]+a-b p$ (obtained from equation (6)) into the expected profit function (equation 5), we can reduce the bivariate objective function into a univariate function of $p$ as follows:

$$
\begin{aligned}
E\left[\Pi\left(Q^{*}(p)\right)\right] & =(p-v) \mu(p)-c_{o} \cdot E\left[Q^{*}(p)-x\right]^{+}-c_{u} \cdot E\left[x-Q^{*}(p)\right]^{+} \\
& =(p-v)(a-b p)-\left[c_{o} \int_{0}^{Q^{*}(p)-a+b p} H(\epsilon) d \epsilon\right]-\left[c_{u} \int_{Q^{*}(p)-a+b p}^{+\infty}[1-H(\epsilon)] d \epsilon\right]
\end{aligned}
$$

where $Q^{*}(p)=H^{-1}\left[\frac{c_{u}}{c_{o}+c_{u}}\right]+a-b p$. Differentiating $E\left[\Pi\left(Q^{*}(p)\right)\right]$ with respect to $p$ gives us the following FOC and second order condition:

$$
\begin{aligned}
\frac{d E\left[\Pi\left(Q^{*}(p)\right)\right]}{d p}= & a-2 b p+b v-\left[c_{o} \cdot H\left(Q^{*}(p)-a+b p\right) \cdot\left(b+\frac{d Q^{*}(p)}{d p}\right)\right] \\
& -E\left[x-Q^{*}(p)\right]^{+}+\left\{c_{u} \cdot\left[1-H\left(Q^{*}(p)-a+b p\right)\right] \cdot\left(b+\frac{d Q^{*}(p)}{d p}\right)\right\} \\
= & a-2 b p-E S\left[Q^{*}(p)\right]+b v \\
\frac{d^{2} E\left[\Pi\left(Q^{*}(p)\right)\right]}{d p^{2}}= & -2 b+\frac{1}{h\left(Q^{*}(p)-a+b p\right)} \cdot \frac{(v-g)^{2}}{(p-g+B)^{3}}
\end{aligned}
$$

where $E S\left[Q^{*}(p)\right]=E\left[x-Q^{*}(p)\right]^{+}$. The sign of the second-order derivative $\frac{d^{2} E\left[\Pi\left(Q^{*}(p)\right)\right]}{d p^{2}}$ depends on the demand distribution and parameter values assumed. If $\frac{d^{2} E\left[\Pi\left(Q^{*}(p)\right)\right]}{d p^{2}}<0$, by setting the first-order condition to zero, we can get

$$
p^{*}=\frac{1}{2 b}\left(a+b v-E S\left[Q^{*}\left(p^{*}\right)\right]\right)
$$

\footnotetext{
${ }^{2}$ The concavity result guarantees the existence of $Q^{*}(p)$. However, the existence of a unique value of $Q^{*}(p)$ requires the assumption that $H(\epsilon)$ is also continuous and differentiable.
} 
It is worth noting that in the additive deterministic demand case (i.e., $x=a+b p$ ), the optimal price is given by the expression $\frac{a+b v}{2 b}$. Since we know that $E S\left[Q^{*}\left(p^{*}\right)\right] \geq 0$, we can conclude that the optimal price in the stochastic setting is always lower than the optimal price in the deterministic setting. Finally, it is also worth noting that Petruzzi and Dada [39] approached the same problem by expressing expected profit as a univariate function of quantity $Q$. They point out that a unique $Q^{*}$ exists for an optimal price $p^{*}$ if the distribution of the random term $\epsilon$ satisfies the inequality $2 r^{2}(\epsilon)+\frac{\partial r(\epsilon)}{\partial \epsilon}>0$ for $\epsilon \in[A, M]$, where $r(\cdot) \equiv h(\cdot) /[1-H(\cdot)]$ and $a-b(v-2 M)+A>0$, where $A(M)$ is the minimum (maximum) possible value of $\epsilon$. The first inequality generalizes the conditions for which optimal solutions to single period newsvendor pricing problems can be identified analytically. Zhan and Shen [51] achieved the same results as above under the linear additive demand form and developed an iterative algorithm and a simulation based algorithm to find the optimal solution. In contrast to the previous papers discussed so far, the authors treated this problem as a nonlinear system with two variables, instead of reducing the bivariate objective function to a univariate function.

In the multiplicative demand case, assume that $x=\mu(p) \epsilon$, where $\mu(p)=a p^{-b}$. Similar to the additive demand setting, $a$ and $b$ are both positive constants with the additional restriction that $b>1, p$ is the market price, and $\epsilon$ is a random variable with a cumulative distribution function $H_{1}(\cdot)$ with a mean of 1 . The expected profit function is again a bivariate function of price and order quantity as given by equation (5). The only difference is that the terms $E[Q-x]^{+}$and $E[x-Q]^{+}$ are redefined as follows (Karlin and Carr [28]):

$$
\begin{aligned}
& E[Q-x]^{+}=\int_{0}^{Q a^{-1} p^{b}} H_{1}(\epsilon) a p^{-b} d \epsilon \\
& E[x-Q]^{+}=\int_{Q a^{-1} p^{b}}^{+\infty}\left[1-H_{1}(\epsilon)\right] a p^{-b} d \epsilon
\end{aligned}
$$

By applying the same procedure as in the additive case, we are able to show that the expected profit function is again concave in $Q$ for a given $p$ and that:

$$
H_{1}\left(Q^{*}(p) a^{-1} p^{b}\right)=\frac{c_{u}}{c_{u}+c_{o}}
$$

After substituting $Q^{*}(p)=H_{1}^{-1}\left[\frac{c_{u}}{c_{u}+c_{o}}\right] a p^{-b}$ (obtained from equation (8)) into the expected profit function equation (5) with the terms $E[Q-x]^{+}$and $E[x-Q]^{+}$redefined as above, we can reduce the bivariate objective function into a univariate function of $p$. Differentiating $E\left[\Pi\left(Q^{*}(p)\right)\right]$ with respect to $p$ gives us the following $\mathrm{FOC}^{3}$ :

$$
\begin{aligned}
\frac{d E\left[\Pi\left(Q^{*}(p)\right)\right]}{d p}= & {\left[1-b\left(1-p^{-1} v\right)\right] a p^{-b} \mu_{\epsilon}+b p^{-1}(v-g) \cdot E L\left[Q^{*}(p)\right]-} \\
& -\left[1-b p^{-1}(p-v+B)\right] \cdot E S\left[Q^{*}(p)\right]
\end{aligned}
$$

\footnotetext{
${ }^{3}$ As before, it is necessary to assume that $H_{1}(\epsilon)$ is continuous and differentiable.
} 
where $E L\left[Q^{*}(p)\right]=\int_{0}^{Q^{*}(p) a^{-1} p^{b}} H_{1}(\epsilon) a p^{-b} d \epsilon$ and $E S\left[Q^{*}(p)\right]=\int_{Q^{*}(p) a^{-1} p^{b}}^{+\infty}\left[1-H_{1}(\epsilon)\right] a p^{-b} d \epsilon$. If $\frac{d^{2} E\left[\Pi\left(Q^{*}(p)\right)\right]}{d p^{2}} \leq 0$, then $p^{*}=\frac{b v}{b-1}+\Delta$, where

$$
\Delta=\frac{a^{-1} b p^{* b}\left[B \cdot E S\left(Q^{*}\left(p^{*}\right)\right)+(v-g) \cdot E L\left(Q^{*}\left(p^{*}\right)\right)\right]}{(b-1)\left(1-a^{-1} p^{* b} \cdot E S\left(Q^{*}\left(p^{*}\right)\right)\right)}
$$

In the multiplicative deterministic demand case (i.e., $x=a p^{-b}$ ), the optimal price is given by the expression $p^{*}=\frac{b v}{b-1}$. Since $\Delta \geq 0$, we can conclude that the optimal price in the stochastic setting is always greater than the optimal price in the deterministic setting. This is in direct contrast to the result for the additive price dependent demand setting. Finally, it is also worth noting that Petruzzi and Dada [39] approached the same problem by expressing expected profit as a univariate function of quantity $Q$. They pointed out that a unique $Q^{*}$ exists provided the distribution of the random term satisfies the inequality $2 r^{2}(\epsilon)+\frac{d r(\epsilon)}{d \epsilon}>0$ for $\epsilon \in[A, M]$ and $b>2$. A possible explanation is also given in their paper regarding the relative magnitudes of riskless and risky optimal prices under different demand forms in terms of demand variance and demand coefficient of variation.

Lau and Lau [32] examined the pricing and ordering policies of a newsvendor facing with a random price-dependent demand under two different objectives, (1) the objective of maximizing the expected profit, and (2) the objective of maximizing the probability of achieving a certain profit level. Two price-demand forms are considered in this paper, an additive form as assumed in our analysis above with a homoscedastic and normally distributed random term (model A), and a relationship fitted with a four-parameter beta distribution based on subjective estimates (model B). In scenario (1A), where model A is considered under objective (1), a numerical example is used to illustrate the effects of price sensitivity and demand variation on the pricing and ordering decisions. The authors showed that lower price sensitivity and demand variation lead to higher optimal price but lower optimal order quantity. In scenario (2A) with zero shortage cost, a counterintuitive result is obtained that more uncertainty in demand may actually increase the probability of achieving a certain profit level. In this paper, numerical solution procedures are provided in each scenario, (1A), (1B), (2A) and (2B), while analytical results are restricted to scenarios (1A) and (2A) with zero shortage cost.

Polatoğlu [40] investigated the joint pricing and ordering decisions under general demand uncertainty, aimed to reveal the fundamental properties independent of demand pattern. Unimodality of the expected profit function that traces the best price trajectory over the order-up-to decision was proved under the assumptions that the mean demand is a monotone decreasing function of price and that the riskless revenue function, which is equal to price times mean demand, is uni-modal. Polatoḡlu [40] also approached the same results proven by Whitin [50], Mills [36] and Karlin and Carr [28], regarding the relative magnitudes of the optimal prices in additive and multiplicative 
models.

Chen, Yan and Yao [17] investigated the price-dependent newsvendor model in a competitive environment. The general demand is stochastic and price-dependent and the firm uses price to compete for the demand. They proved and showed the conditions for the existence of the purestrategy Nash equilibrium and its uniqueness. Raising individual prices at any equilibrium of the game could increase the total system profit, but no single firm would follow this strategy since it has already optimized its own profit conditioned on the other firms' pricing schemes. At the cooperative equilibrium, each self-interested firm has an incentive to lower its price to grab more demand. They concluded that competition leads to a lower equilibrium price and higher stock levels in all firms.

Arcelus, Kumar and Srinivasan [5] studied the pricing and ordering policies of a profit-maximizing newsvendor that faces trade incentives offered by the supplier (i.e., wholesale price discount to the newsvendor and direct rebate to the end customers) and stochastic price-dependent demand. Let $(v, R)$ denote the supplier's pricing/rebate policy, where $v$ is the wholesale price and $R$ is the direct rebate to end customers. Suppose the original wholesale price is $v_{0}$. The newsvendor receives an offer $\left(v_{0}-d, 0\right)$ under the price discount policy and $\left(v_{0}, R\right)$ under the direct rebate policy. No specific functional form is assumed for the deterministic component of the demand and both additive-error and multiplicative-error demands are considered in this paper. The authors derived the demand conditions under which the results obtained in Petruzzi and Dada [39] regarding the relative magnitude of the retail prices in riskless and risky cases still hold in this setting. The effectiveness of the two trade incentives on the newsvendor's decisions is then measured and contrasted by the passthrough ratio, defined as $\delta=\frac{d p}{d v}$, under the price-discount policy, and the claw-back ratio, defined as $\lambda=\frac{d p}{d R}$, under the direct-rebate policy. They showed analytically that the pass-through ratio is always greater than the claw-back ratio for any deterministic demand and that the claw-back ratio in the risky case is smaller than that in the riskless case for demand forms with additive-error and linear deterministic component. It is worth noting that negative claw-back ratio is possible, that is, the newsvendor may offer a lower retail price to the end customers in spite of the rebate the consumers have already received from the supplier. However, no further analytical results are obtained concerning the impact of demand uncertainty on the newsvendor's optimal order quantity in this setting. Numerical analysis is conducted under the assumption of uniformly distributed error term. Observations include the dominance of the pass-through ratio over the claw-back ratio in the risky case and the improved newsvendor's expected profits under either trade incentive. 


\subsection{Demand and Marketing Effort}

Customer demand can often be influenced by a number of marketing activities (e.g., advertising, sales calls, and in-store displays). Kraiseburd, Narayanan and Raman [31] studied a model that incorporates such an aspect in the newsvendor scenario. Their setting is as follows. Let $e$ represent the marketing "effort" expended by the buyer. Mean customer demand is modeled as a nondecreasing function of $e$ (i.e., $\frac{d \mu(e)}{d e} \geq 0$ and $\frac{d^{2} \mu(e)}{d e^{2}}<0$ ) while the variance in customer demand (i.e., $\left.\sigma^{2}\right)$ is assumed to be independent of the marketing effort $e$. $c(e)$ represents the cost per unit of effort and is assumed to be convex in $e$. In this case, the optimal marketing effort for the firm (i.e., $\left.e^{*}\right)$ can be determined by solving the following FOC:

$$
(p-v) \frac{d \mu(e)}{d e}-\frac{d c(e)}{d e}=0
$$

The optimal order quantity $Q^{*}$ can then be found by setting $F\left(Q^{*}\right)=\frac{p-v+B}{p-g+B}$ where the cumulative distribution function of demand ${ }^{4}$ has a mean $\mu\left(e^{*}\right)$.

The analysis presented above could also be easily extended to a situation where marketing effort affects demand in a way that demand variance decreases as more effort is made in the selling season. Gerchak and Mossman [22] examined the effects of demand randomness on optimal order quantities and the associated expected costs by applying mean-preserving transformations to the demand variable. They considered the family of random variables $x_{\alpha}$ representing demand as follows:

$$
x_{\alpha}=\alpha x+(1-\alpha) \mu
$$

where $\alpha \geq 0$ is an arbitrary constant. Obviously, $E\left(x_{\alpha}\right)=\mu$ for all $\alpha$. We note that if for some constants $\alpha_{1}>\alpha_{2}$, then $x_{\alpha_{1}}$ is more variable than $x_{\alpha_{2}}$ and using this property the authors are able to show that:

$$
\begin{aligned}
Q_{\alpha}^{*} & =\alpha Q^{*}+(1-\alpha) \mu \\
C_{\alpha}\left(Q_{\alpha}^{*}\right) & =\alpha C\left(Q^{*}\right)
\end{aligned}
$$

where $C\left(Q^{*}\right)$ represents the expected cost associated with an optimal order quantity of $Q^{*}$ under demand $x$, and $C_{\alpha}\left(Q_{\alpha}^{*}\right)$ represents the expected cost associated with an optimal order quantity $Q_{\alpha}^{*}$ under demand $x_{\alpha}$.

\footnotetext{
${ }^{4}$ For example, if demand is normally distributed with mean $\mu\left(e^{*}\right)$ and standard deviation $\sigma$, then $Q^{*}=\mu\left(e^{*}\right)+z \sigma$ where $z$ is the unit normal deviate such that $F(z)=\frac{p-v+B}{p-g+B}$.
} 
To analyze the case in which demand uncertainty is affected by marketing effort, replace $\alpha$ with $\alpha(e)$ in the above three equations. Assume that $\alpha(e)$ is a monotone decreasing function of $e$ and that $\alpha(0)=1$, and $\alpha(+\infty)=0$. The equation $\alpha(+\infty)=0$ simply says that demand uncertainty may be eliminated by tremendous marketing effort, which is impossible in practice due to the unbearable cost of those marketing activities. Also assume that $c(0)=0$.

After substituting and rearranging, we obtain

$$
Q_{\alpha\left(e^{*}\right)}^{*}=\alpha\left(e^{*}\right) Q^{*}+\left(1-\alpha\left(e^{*}\right)\right) \mu
$$

Consider the relative magnitudes of $Q_{\alpha\left(e^{*}\right)}^{*}$ and $Q *$. As

$$
\frac{d Q_{\alpha(e)}^{*}}{d e}=\frac{d \alpha(e)}{d e}\left(Q^{*}-\mu\right)
$$

the optimal order quantity increases in marketing effort $e$ if and only if $Q^{*}<\mu$, i.e., $F(\mu)>\frac{p-v+B}{p-g+B}$.

The maximum expected profit for a specific marketing effort $e$ can be expressed as

$$
\begin{aligned}
E\left[\Pi\left(Q_{\alpha(e)}^{*}, e\right)\right] & =(p-v) \mu-C_{\alpha(e)}\left(Q_{\alpha(e)}^{*}, e\right)-c(e) \\
& =(p-v) \mu-\alpha(e) C\left(Q^{*}\right)-c(e)
\end{aligned}
$$

Differentiating $E\left[\Pi\left(Q_{\alpha(e)}^{*}\right)\right]$ with respect to $e$, we get

$$
\begin{aligned}
\frac{d E\left[\Pi\left(Q_{\alpha(e)}^{*}\right)\right]}{d e} & =-\frac{d \alpha(e)}{d e} C\left(Q^{*}\right)-\frac{d c(e)}{d e} \\
\frac{d^{2} E\left[\Pi\left(Q_{\alpha(e)}^{*}\right)\right]}{d e^{2}} & =-\frac{d^{2} \alpha(e)}{d e^{2}} C\left(Q^{*}\right)-\frac{d^{2} c(e)}{d e^{2}}
\end{aligned}
$$

If $\frac{d^{2} E\left[\Pi\left(Q_{\alpha(e)}^{*}\right)\right]}{d e^{2}}<0$, we can derive the optimal marketing effort $e^{*}$ by setting the partial derivative $\frac{d E\left[\Pi\left(Q_{\alpha(e)}^{*}\right)\right]}{d e}$ equal to zero, which determines the optimal order quantity of the buyer. Clearly, the second-order condition holds for a concavely decreasing $\alpha(e)$ and a convexly increasing $c(e)$. In this case, $e^{*}$ can be characterized by the following equation:

$$
\frac{d \alpha(e)}{d e} C\left(Q^{*}\right)+\frac{d c(e)}{d e}=0 .
$$

For this setting (i.e., demand is influenced by marketing effort), our analysis indicates that an increase (decrease) in mean demand (with demand variability unchanged) due to marketing effort leads to an increase (decrease) in the optimal stocking quantity $Q^{*}$. On the other hand, the impact of a decrease in demand variability (with mean demand unchanged) due to marketing effort is not as clear. However, we can identify parametric conditions under which a decrease in demand variability due to marketing effort will lead to increases in the optimal stocking quantity $Q^{*}$. 


\subsection{Demand and Stocking Quantity}

Marketing researchers often argue that quantity displayed can have a positive impact on demand and thus, ultimate sales. In essence two types of stimuli that impact demand positively due to the quantity displayed are: (a) a selective effect; and (b) an advertising effect. There is a significant body of literature on inventory systems where demand is positively impacted by stock level. These stock dependent inventory models developed so far can be classified into two categories. Models in the first category dealt with demand that is a function of the initial stock level, while the others assumed that demand is dependent on the instantaneous stock level and may thus vary over time. We will focus on newsvendor models with stock-dependent demand. Readers who are also interested in other inventory systems may refer to Urban [45] for a comprehensive review of the stock-level-dependent periodic-review models.

Baker and Urban [8] studied a deterministic single-period model in which demand at time $t$, $x(t)$, is a polynomial function of the instantaneous stock level, $i(t)$, and solved for the optimal order quantity $(Q)$, which is also the initial stock level, under the objective of maximizing the total profit. Specifically, they assumed a demand function $x(t)=a[i(t)]^{1-b}$, where $a>0,1>b>0$. The constant parameter $b$ is constrained to be some value between zero and one to ensure that the marginal increase in demand decreases as the stock level increases. Sensitivity analysis conducted in this paper showed that failing to consider the stimulating effect of stock on demand when it exists may lead to miserable optimization results, since the model is quite sensitive to changes in $b$. Urban and Baker [46] investigated a similar deterministic newsvendor problem with stockdependent, price sensitive and time proportional demand. They expressed the demand rate as $x(p, t, i)=a p^{-k} t^{r-1} i^{1-b}$, where $a>0, k>0, r>0$ and $1>b>0$. Although no structural results were obtained for the general case, sensitivity analysis performed regarding the effects of price, time and stock level on demand reassured the importance of recognizing the correct demand pattern.

Urban [44] also examined a stock-dependent newsvendor model. Demand in this paper takes the same polynomial function as in Baker and Urban [8], but with the total stock level $i(t)$ replaced by the observed stock level $\varphi(t)$ assuming that customers are only influenced by the displayed products. That is, $x(\varphi(t))=a[\varphi(t)]^{1-b}, a>0,1>b>0$, where $\varphi(t)=\min (s, i(t))$ and $s$ denotes the shelf space allocated to that product. With the further assumption of the quasi-concavity of the profit function, Urban derived the closed form expressions for the optimal order quantity $Q^{*}$, which is equal to $i^{*}(0)$, and the optimal shelf space $s^{*}$ and investigated the interdependency between the two decisions. The relationship between $Q^{*}$ and $s^{*}$ can be characterized by the equation $\frac{s^{*}}{Q^{*}}=\frac{(1-b)(p-c)}{(1-b)(p-c)+v}$, where $v$ is the unit shelf-space cost. Urban also pointed out that the holding cost, which can be considered as the negative salvage cost in our setting, has an equal impact on 
the optimal values of the two decision variables.

Most of the inventory models where demand is assumed to be a function of instantaneous stock level seldom incorporate the uncertainty of demand due to technical complexity. Similarly, when stochastic stock-dependent demand is considered in an inventory system, researchers usually interpret the word "stock" as "initial stock", which can be viewed as "order quantity" in a single period newsvendor model. In the rest of the section, some simple results will first be derived for a special case of the stochastic newsvendor model that uses initial stock level as an independent variable, followed by a brief literature review of stochastic stock-dependent newsvendor models.

Assume that customer demand is linearly increasing in the buyer's stocks as follows ${ }^{5}: x(Q)=$ $a+b Q+\epsilon, a>0,0<b<1$, where $\epsilon$ is a random variable with probability density function $h_{2}(\cdot)$ and cumulative distribution function $H_{2}(\cdot)^{6}$. Assuming that the buyer is risk neutral and would like to maximize the expected profit, the objective function for this setting is as follows:

$$
E[\Pi(p, Q)]=(p-v)\left(a+b Q+\mu_{\epsilon}\right)-c_{o} \cdot E[Q-x(Q)]^{+}-c_{u} \cdot E[x(Q)-Q]^{+}
$$

where

$$
\begin{aligned}
E[Q-x(Q)]^{+} & =\int_{0}^{(1-b) Q-a} H_{2}(\epsilon) d \epsilon \\
E[x(Q)-Q]^{+} & =\int_{(1-b) Q-a}^{+\infty}\left[1-H_{2}(\epsilon)\right] d \epsilon
\end{aligned}
$$

It is quite straightforward to show that the expected profit function is concave in $Q$. By equating the first order derivative of the objective function with respect to $Q$ to zero, we obtain

$$
H_{2}\left((1-b) Q^{*}-a\right)=\frac{\frac{p-v}{1-b}+B}{p-g+B}
$$

Note that $H_{2}((1-b) Q-a)=P((1-b) Q-a \leq \epsilon)=P(Q \leq x(Q))$. Since $0<b<1$, the fractile value given by $\frac{\frac{p-v}{1-b}+B}{p-g+B}$ is greater than the critical fractile value $\frac{p-v+B}{p-g+B}$ for the classic newsvendor model. Hence, we can conclude that the stock-dependent demand in an additive form causes an increase in the order quantity as compared to the classic newsvendor model.

Dana and Petruzzi [19] investigated an extension of the classic newsvendor model where each customer is interested in maximizing her own expected utility and chooses whether to visit the firm or go for an outside option, $u$, based on the firm's price and initial stock level she observes

\footnotetext{
${ }^{5}$ The assumption of linearity is made for tractability but the results hold as long as demand is non-decreasing in quantity.

${ }^{6}$ The mean of the random term $\epsilon$ is not required to be zero for the linear additive demand form.
} 
as well as her own valuations of the product and the outside option. Given these assumptions of consumer behavior, the firm faces uncertain aggregate demand which can be modeled as a multiplicative function of both its price and its initial stock level. The authors considered two cases: the exogenous price case for a firm that is merely a price taker and the endogenous price case where price can be chosen optimally. Both the benchmark policy, which ignores the effect of stock on demand, and the optimal policy are determined in each case. The uniqueness of the optimal policies again requires specific restrictions on the distribution of consumers' outside options and/or the distribution of aggregate demand as mentioned in the previous section. The result of a comparison between the optimal policy and its corresponding benchmark policy is that if a firm internalizes the effect of stock on demand, it usually holds more stock, provides higher service level and earns higher expected profit no matter it is a price taker or price setter. It is also worth mentioning that sequential optimization of price and stocking quantity can be applied in the endogenous price case.

Balakrishnan, Pangburn and Stavrulaki [9] generalized the newsvendor model to incorporate the stochastic and initial-stock-level dependent demand. The authors proposed a general stochastic demand model via an inverse fractile function to capture the stimulating effect of stock on demand. Let $f(Q, x)$ denote the probability density function (pdf) of $x(Q)$ and $l(Q)$ denote the stockdependent lower support of the pdf. The corresponding inverse fractile function is defined as $\phi(Q, \rho)=F^{-1}(Q, \rho)$, where $\rho$ is some given fractile value between 0 and 1 . To ensure that demand increases at a decreasing rate with the initial stock level, $\phi(Q, \rho)$ is assumed to be a concavely increasing function of $Q$, which is later shown to be the sufficient condition for the concavity of the profit function. Applying a variable transformation $x=\phi(Q, \rho)$, the expected sales for a given order quantity $Q$ can be expressed as $S(Q)=\int_{0}^{F(Q, Q)} \phi(Q, \rho) d \rho+Q(1-F(Q, Q))$. The marginal expected sales $S^{\prime}(Q)$ was found to consist of two parts, $1-F(Q, Q)$ and $\int_{0}^{F(Q, Q)} \phi^{\prime}(Q, \rho) d \rho$. The former was interpreted as the availability effect of stocking higher quantities and the latter as the promotional effect of stocking more. When the stock stimulating effect is ignored, the term representing the promotional effect drops out in the optimality condition. By observing so, the authors extended, from a multiplicative demand form to a general demand form, the conclusion reached in Dana and Petruzzi [19] that the consideration of the demand-stimulating effect of stock leads to higher initial stock levels and higher fill rates. 


\section{Supplier Pricing Policies and Stocking Quantity}

Quantity discounts are a wildly adopted pricing scheme in practice. Basically, the notion is that the larger the order quantity, the lower the unit procurement cost and the justification for its popularity includes, but not limited to, costs transfer, price discrimination and channel coordination. When a supplier has a high fixed cost, s/he may choose to offer quantity discounts to encourage larger orders, which helps achieve economies of scale. Given that the risk of larger orders is mainly carried by the buyer, suppliers are often more than willing to practice quantity discounts when inventory costs are high. As for price discrimination, quantity discounts helps suppliers to identify price-insensitive retailers from price-sensitive ones and charge higher prices (if possible) to retailers who are willing to pay more. In this area, there are two interrelated streams of literature which are of relevance: (a) using different supplier pricing policies to coordinate a supply chain; and (b) modifying the traditional newsvendor analysis to handle different types of supplier pricing policies. Research contributions in each area are discussed in this section.

\subsection{Coordination in a Supply Chain through supplier pricing policies}

Consider a supply chain (SC) with a single supplier (S) and a single newsvendor-type retailer (R) facing uncertain demand. Assume that the two channel members have full information about demand and each other's cost structure and that the retailer places an order at the beginning of the selling season. Then, the retailer's expected profit $\left(E\left[\Pi_{R}(Q)\right]\right)$ can be expressed as

$$
E\left[\Pi_{R}(Q)\right]=(p-v) \mu-(p-v+B) E[x-Q]^{+}-(v-g) E[Q-x]^{+}
$$

and based on the order placed by the retailer $(Q)$ and a supplier variable production cost $(c)$, the supplier's expected profit $\left(E\left[\Pi_{S}\right]\right)$ is:

$$
E\left[\Pi_{S}\right]=(v-c) Q
$$

respectively. Since the supplier will not operate with negative or zero profit, it is reasonable to assume $v>c$. The expected supply chain profit $\left(E\left[\Pi_{S C}\right]\right)$, which is the sum of the retailer's and supplier's profits, is

$$
E\left[\Pi_{S C}\right]=(p-c) \mu-(p-c+B) E[x-Q]^{+}-(c-g) E[Q-x]^{+}
$$

When the channel members are only interested in maximizing their own profits, the retailer will

place an order of $F^{-1}\left(\frac{p-v+B}{p-g+B}\right)$ units, which is smaller than the order quantity $F^{-1}\left(\frac{p-c+B}{p-g+B}\right)$ that 
maximizes the channel profit. Hence, offering some form of a quantity discount to the retailer, which results in a lower wholesale price, can be a feasible solution to achieving channel coordination. However, it must be made clear that not all forms of quantity discounts can help obtain channel coordination.

According to Jeuland and Shugan [26], among various quantity discounts, only those that make channel members' profit maximizing incentives consistent with overall channel profit maximization can coordinate the supply chain. This implies that channel members' profit functions should be some fixed linear function of the channel profit under the channel-coordinating quantity discount. Define $x(p)$ as a price-dependent demand function such that demand is decreasing in price (thus, $p(x)$ is the inverse of $x(p)), k_{1}\left(0<k_{1}<1\right)$ as some fraction of total supply chain profits received by the supplier (hence, the retailer receives $1-k_{1}$ ), $k_{2}$ as some fixed amount of total supply chain profit allocated to the supplier, and $c(C)$ as the variable per unit cost for the supplier (retailer). Based on this, one possible form of a quantity discounted price $v(x)$ which can coordinate the supply chain is $v(x)=k_{1}(p(x)-C-c)+\left(\frac{k_{2}}{x}\right)+C$.

Ingene and Parry [24] showed the existence of a two-part tariff wholesale pricing policy that coordinates a supply chain with a single supplier and multiple independent retailers. It is assumed that the deterministic demand faced by each retailer is unique and concavely decreasing in retail price. The number of retailers who choose to participate (by obtaining non-negative profits) can also be considered as a decision in this problem. Let $v\left(Q_{i}\right)$ denote the unit payment by retailer $i$ for an order of size $Q_{i}$ and $c$ denote the supplier's marginal cost. Utilizing marginal analysis techniques, the authors showed that a two-part tariff ${ }^{7}$ in which $v\left(Q_{i}\right)=c+\phi_{c} / Q_{i}$ coordinates the supply chain, where $\phi_{c}$ is a fixed fee paid by each participating retailer to the supplier. The number of participating retailers under this type of pricing policy, denoted by $N_{c}^{*}$, should be no more than the number of participating retailers in the integrated system since each retailer in the decentralized system has a non-negative $\phi_{c}$ to cover. Finally, it is shown that this type of coordinating two-part tariff policy is different from the two-part tariff policy that maximizes the supplier's profit except in the following two cases: the supply chain consists of a single retailer, or the sales of the retailer that achieves exactly zero profit from participation is equal to the total sales divided by the number of participating retailers.

Weng [48] studied the coordination issues of a single-supplier-single-distributor system under price-dependent stochastic demand. A two-part tariff policy, a fixed payment plus the wholesale price, was shown to be sufficient to achieve the coordination. The author assumed that all demand

\footnotetext{
${ }^{7} \mathrm{~A}$ two-part tariff can be considered a special case of a quantity discount where for any quantity purchased, the retailer is required to pay a fixed fee plus a per unit fee.
} 
must be satisfied and a second order is allowed at a higher cost when demand exceeds the initial order quantity. Decisions to be made include the distributor's selling price and initial order quantity as well as the supplier's unit profit margin. The supplier's selling price is equal to the sum of the unit profit margin and the unit production cost. In the absence of coordination, it is assumed that the supplier may not know the distributor's actual response to its wholesale price and thus determines the unit profit margin based on its estimated order quantity, which may deviate from the distributor's actual order quantity. When coordination is enabled, the supplier has complete information about the distributor's response. The price-dependent random demand is modeled by a generalized phase-type distribution, i.e., $F(x \mid p)=1-e^{-x / \mu(p)} \sum_{n=1}^{+\infty} a_{n} \sum_{i=0}^{n-1} \frac{(x / \mu(p))^{i}}{i !}$, where $a_{n}, n=1,2, \ldots, \infty$ is some appropriately-chosen discrete probability distribution and $\mu(p)=d p^{-k}$, $d>0, k>1$. The parameter $k$ represents the sensitivity of demand to price. The optimal pricing and ordering policies with and without coordination are then characterized and constrasted under previous assumptions. It is shown analytically that the optimal initial order quantity with coordination is always greater than the optimal initial order quantity without coordination and the gap increases in $k$. Two effects of coordination are found to contribute to the above result, the reduction in the unit overage and underage costs and the reduction in the supplier's selling price. Numerical analysis demonstrated the necessity of coordination for both parties when demand is highly sensitive to the distributor's selling price. The author also developed a two-part tariff policy to coordinate the system in which the supplier sets its selling price equal to its unit production cost and the distributor makes a fixed payment to the supplier.

In Weng [49], a generalized newsvendor model was developed to examine the effect of coordination for a single-supplier-single-retailer supply chain under stochastic demand and an all-units quantity discount was designed to coordinate the system. The only decision included in this model is the buyer's order size. No specific distribution is assumed for the demand. Unfilled demand may be backordered at a cost and a second order may be placed to the supplier depending on whether the additional order generates positive profits for both parties. Let $b$ denote the unit backorder cost, $t_{2}$ denote the ordering cost for the second order and $s_{2}$ be the supplier's setup cost for the second run. The buyer is willing to place a second order if the unfilled demand, $q$, satisfies the inequality $(p-v-b) q-\left(t_{2}+s_{2}\right)+B q \geq 0$, where the sum of the first two terms is the profit to be obtained from placing a second order and the last term is the shortage cost incurred if a second order is not placed (recall that $B$ is the per unit shortage/lost sales cost). This modeling of unfilled demand can be used to cover situations in which all demand must be satisfied by setting $B \rightarrow+\infty$ or no re-supply is available from the supplier by letting $b=p-v+B$. Analytically, the author showed that, for an increasing concave demand distribution function, the supply chain's optimal order quantity $\left(Q_{S C}^{*}\right)$ is always larger than the retailer's optimal order quantity, $Q_{R}^{*}$ when 
coordination is not implemented. Let $c_{\min }\left(Q_{S C}^{*}, \rho\right)$ (where $\rho$ is some non-negative constant) denote the supplier's lowest acceptable per unit price $v$ satisfying $E\left[\Pi_{S}\left(Q_{S C}^{*}\right)\right] \geq(1+\rho) E\left[\Pi_{S}\left(Q_{R}^{*}\right)\right]$, where $E\left[\Pi_{S}(\cdot)\right]$ refers to the supplier's expected profit with a particular order quantity. Let $c_{\max }\left(Q_{R}^{*}, \gamma\right)$ (where $\gamma$ is some non-negative constant) denote the retailer's highest acceptable per unit price $v$ satisfying $E\left[\Pi_{R}\left(Q_{S C}^{*}\right)\right] \geq(1+\gamma) E\left[\Pi_{R}\left(Q_{R}^{*}\right)\right]$. If $c_{\max }\left(Q_{R}^{*}, \gamma\right) \geq c_{\min }\left(Q_{S C}^{*}, \rho\right)$, the author argued that an all-units quantity discount in which the single price break quantity and the corresponding unit price be set to $Q_{S C}^{*}$ and $c_{\max }\left(Q_{R}^{*}, \gamma\right)$, respectively, can coordinate the system since both parties are no worse off. The two parameters $\rho$ and $\gamma$ can be interpreted as specifying the allocation of the increased system profit due to coordination. For example, if $\rho=0$, then all increases in supply chain profits are allocated to the buyer, while if $\gamma=0$, then all increases in supply chain profits are allocated to the supplier.

Cachon and Lariviere [16] focused on the use of revenue-sharing contracts in supply chain coordination and offered comparisons between revenue-sharing and other contracts including quantity discounts. Again, consider a single-supplier-single-retailer supply chain. The profit-maximizing retailer makes two decisions, order quantity and retail price (shortage cost is not included). Let $S(Q, p)$ be the expected sales for the quantity-price pair $(Q, p), c$ be the unit production cost of the supplier and $C$ be the retailer's unit operating cost. The retailer's expected revenue can then be expressed as $R(Q, p)+g Q$, where $R(Q, p)=(p-g) S(Q, p)$, and the expected system profit $E\left[\Pi_{S C}(Q, p)\right]=R(Q, p)-(c+C-g) Q$. Let $\left(Q^{*}, p^{*}\right)$ denote the quantity-price pair that maximizes the expected system profit. The authors demonstrated that, for a fixed retail price $p^{*}$, the quantity discount scheme $v(Q)=(1-\alpha) \frac{R\left(Q, p^{*}\right)}{Q}+\alpha(c+C)+(1-\alpha) g-C$ can also coordinate the supply chain. However, this specific quantity discount may fail to coordinate the system when the retail price deviates from $p^{*}$, while the linear relationship between the retailer's expected profit and the system's expected profit maintains under revenue sharing. Furthermore, as $R(Q, p)$ is included in $v(Q)$, quantity discounts may only apply to a multi-retailer situation where the retailers have the same revenue function.

\subsection{Alternative pricing policies and the newsvendor analysis}

Three common types of quantity discount supplier pricing are:

- Linear quantity discount (LD). A linear discount scheme assumes that the supplier offers a per unit price $v^{L D}(Q)$ decreasing in the quantity $Q$ ordered by the retailer. The specific linear discount scheme we consider is $v^{L D}(Q)=v_{f}-t Q$ where $t$ is some per unit constant discount rate, and $v_{f}$ is the base price. 
- All-units (AU) quantity discount. As with the linear discount scheme, under the all-units quantity discount policy the supplier also offers a per unit price $v^{A U}(Q)$ which is declining in the quantity $Q$ ordered by the retailer. The key difference is that the price per unit decline is a step function and is specified as follows. Define $w_{i}(i=1, \ldots, n)$ as alternative per unit prices such that $w_{1}>w_{2}>\ldots>w_{n}$ and corresponding maximum quantities to each per unit price as $q_{i+1}(i=1, \ldots, n)$ such that $q_{1}<q_{2}<\ldots<q_{n}$ (in general, $q_{1}$ is assumed to be 0 ). Then under the all-units discount scheme, the per unit price charged by the supplier is: $w_{i}$ for $q_{i}<Q \leq q_{i+1}(i=1, \ldots, n)$ where $Q$ is the quantity ordered by the retailer.

- Incremental-units (IU) quantity discount. In comparison to the all-units quantity discount scheme, under the incremental-units policy the price quoted by the supplier only applies to incremental units ordered by the retailer. Hence, under this pricing scheme, the per unit average price charged by the supplier is: $w_{1}$ for $q_{1}<Q \leq q_{2},\left(w_{1} q_{2}+w_{3}\left(Q-q_{2}\right)\right) / Q$ for $q_{2}<Q \leq q_{3},\left(w_{1} q_{2}+w_{3}\left(q_{3}-q_{2}\right)+w_{3}\left(Q-q_{3}\right)\right) / Q$ for $q_{3}<Q \leq q_{4}$, and so on.

The cost per unit $\left(v^{L D}(Q), v^{A U}(Q)\right.$, and $\left.v^{I U}(Q)\right)$ and the total cost of each policy is illustrated in Figure 1. Assuming the retailer operates as a newsvendor, the impact of each of these schemes on the optimal order quantity for each of these schemes is as follows.

\section{Insert Figure 1 about here}

Assuming a risk-neutral retailer facing with a linear quantity discount, the retailer's expected profit function can be attained by simply replacing $v$ with $v(Q)=v_{f}-t Q$ in its expected profit function in the basic case.

$$
E\left[\Pi_{R}\right]=(p-v(Q)) \mu-(p-v(Q)+B) E[x-Q]^{+}-(v(Q)-g) E[Q-x]^{+}
$$

By imposing certain parametric restrictions ${ }^{8}$, the optimal order quantity $Q_{L D}^{*}$ is characterized as follows:

$$
F\left(Q_{L D}^{*}\right)=\frac{p-v_{0}+B}{p-g+B}+\frac{2 t Q}{p-g+B}
$$

Given that $\frac{2 t Q}{p-g+B}>0$, it is obvious that the buyer would be induced to order more under linear discounting scheme as compared to the fixed price scheme. While a closed form expression for $Q_{L D}^{*}$ cannot be derived for an arbitrary demand distribution, the optimal value of $Q_{L D}^{*}$ can be found via a simple uni-dimension search algorithm.

\footnotetext{
${ }^{8}$ These restrictions are: $0<t<\min \left[0.5 f(Q)(p-g+B),(v-g) Q^{-1}\right]$ for all possible values of $Q$.
} 
To solve the buyer's optimal order quantity decision $\left(Q^{*} A U\right)$ assuming that the supplier offers an all-units quantity discount, the algorithm in Figure 2 can be used. To solve the buyer's optimal order quantity decision $\left(Q^{*} I U\right)$ assuming that the supplier offers an incremental quantity discount, the algorithm shown in Figure 3 can be used. $^{9}$

Insert Figures 2 and 3 about here

Jucker and Rosenblatt [27] studied the implications of quantity discounts for both the retailer and supplier in the context of the newsvendor problem. Consider a profit maximizing retailer that faces deterministic demand and an all-units quantity discount offered by the supplier. The authors showed that the retailer orders up to $d_{i}$ units if the amount of units she must purchase, denoted by $Q^{\prime}$, falls into the interval $\left[d_{i}, q_{i+1}\right)$, and orders exactly $Q^{\prime}$ units if $Q^{\prime}$ is in $\left[q_{i}, d_{i}\right)$, where $d_{i}=q_{i+1} \frac{w_{i+1}+g}{w_{i}+g} \cdot d_{i}$ can be interpreted as the breakeven point at which the marginal cost remains the same between ordering $d_{i}$ and $q_{i+1}$ units. Since this kind of behavior reflects zero marginal cost of units in the interval $\left[d_{i}, q_{i+1}\right)$, the all-units quantity discount can be characterized by a marginal profit function which equals $w_{i}$ for $q_{i} \leq Q<d_{i}$ and 0 for $d_{i} \leq Q<q_{i+1}$. Incremental quantity discounts can be modeled as a special case where $d_{i}=q_{i+1}$. Using marginal analysis, the authors then proposed a new solution procedure for newsvendor problems with all-units quantity discount. Traditionally, the global maximum is found through the algorithm outlined earlier. However, the authors suggested that, for cases in which the marginal profit of an additional unit equals $w_{i}$ cannot be solved analytically, it be better to first compare the values of the marginal cost function and marginal revenue function from the lowest price break quantity to locate the intersections if exist. While price break quantities can be candidates for the global optimum in the case of all-units quantity discount, the paper mentioned that we only need to pay attention to the intersections when an incremental quantity discount is considered.

Pantumsinchai and Knowles [37] presented solution algorithms to the standard container size discounts in the newsvendor environment. The newsvendor determines the optimal order quantity and the number of different standard containers to make up the order under random demand. Suppose that there are $m$ standard container sizes available with $q_{1}>q_{2}>\ldots>q_{m}$. The unit purchase costs associated with container sizes satisfy the inequality $0<p_{1} \leq p_{2} \leq \ldots \leq p_{m}$. Let $I$ denote the newsvendor's initial inventory level and $y_{i}$ denote the optimal order-up-to level that minimizes $G\left(y_{i}, I\right)$, the purchase cost if applicable plus the expected overage and underage cost when only the $i$ th container size is available. Define $n_{i}=\left(y_{i}-I\right) / q_{i}, i=1,2, \ldots, m$. The authors showed

\footnotetext{
${ }^{9}$ The process is similar to that of the all-units quantity discount except that in this case, we only compute the expected profit when order quantities are in the feasible range.
} 
that the newsvendor should not order when $I \geq y_{1}$. In the case where $I<y_{1}$, the optimal order quantity $Q^{*}$ is bounded by $n_{u} q_{u}$ and $\left(n_{u}+1\right) q_{u}$, where $n_{u}=\left(y_{u}-I\right) / q_{u}$ and $u$ is chosen such that $n_{i}=0, i=1, \ldots, u-1$ and $G_{1}\left(\left(n_{1}+1\right) q_{1}+I, I\right) \geq G_{2}\left(\left(n_{2}+1\right) q_{2}+I, I\right) \geq \ldots \geq G_{u}\left(\left(n_{u}+1\right) q_{u}+I, I\right)$. Solution algorithms are then proposed for solving the general case and the restricted case where the newsvendor is required to use successively smaller container sizes. In addition, when a fixed cost $K$ is incurred for placing an order, the authors suggested comparing $G\left(y^{*}, I\right)+K$ with $G(I, I)$ and ordering if $G(I, I)$ is larger.

Lin and Kroll [35] considered both all-units quantity discount and incremental discount in the newsvendor problem with dual performance measures: "maximize the expected profit subject to a constraint that the probability of achieving a target profit level is no less than a predetermined risk level". The retailer's ordering decision was analyzed under four models: Model 1: all-units discount with zero shortage cost; Model 2: incremental discount with zero shortage cost; Model 3: all-units discount with positive shortage cost; and Model 4: incremental discount with positive shortage cost. For Model 1, using results from Lau [34], the authors showed that the constraint $\operatorname{Prob}(\Pi \geq T) \geq \theta$, where $\Pi$ is the actual profit, $T$ is the target profit level, and $\theta$ is the predetermined risk level, actually imposes another feasible condition on $Q$ associated with $w_{i}$, i.e., $T /\left(p-w_{i}\right) \leq$ $Q \leq\left((p-g) F^{-1}(1-\theta)-T\right) /\left(w_{i}-g\right)$. Therefore, the feasible interval for $Q$ corresponding to $w_{i}$ can be obtained by combining this interval with the interval $\left[q_{i}, q_{i+1}\right)$ in this case. The optimal order quantity can be found by solving the newsvendor model with an all-units quantity discount scheme using the algorithm described earlier. For Model 2, the decision variable $Q$ has the same feasible interval as for Model 1 for each $w_{i}$ and closed-form expression for the optimal solution can be derived. For the other two models with positive shortage cost, again using the results from Lau [34], the constraint on the risk level can be replaced by the inequality $F\left(D_{2 i}-F\left(D_{1 i}\right)\right) \geq \theta$, where $D_{2 i}=\left[T+\left(w_{i}-g\right) Q_{i}\right] /(p-g)$ and $D_{1 i}=\left[\left(p-v_{i}+B\right) Q_{i}-T\right] / B$ where $Q_{i}$ is the feasible order quantity in the interval $\left[q_{i}, q_{i+1}\right]$. Since the constraint is nonlinear, no closed form solution can be obtained in these two cases.

Burnetas, Gilbert and Smith [14] addressed a pricing-policy design problem of a profit-maximizing supplier that has less demand information than the newsvendor-type buyer does. The optimal all-units discount and the optimal incremental discount were also contrasted in terms of the effectiveness of increasing the supplier's expected profit in this paper. The authors modeled information asymmetry by considering $n$ buyer types that have different demand distribution $F_{i}(x)$, $i=1,2, \ldots, n$, but the same cost structure. For the supplier, each type $i$ has a probability of $p_{i}$ to be the real type of the buyer. And it is assumed that $F_{j}(x)$ stochastically dominates $F_{i}(x)$ for 
$j>i^{10}$. The buyer has an outside supply source to purchase the products at a price of $u, c<u<p$. In such an environment, following the revelation principle, the pricing mechanism design problem can be formulated as maximizing the supplier's expected profit subject to constraints that each buyer type prefers the quantity-price option intended for him and accepting the intended option should yield at least as much expected profit as resorting to the outside supply source. The solution to this problem is referred to as the fixed-package pricing policy (i.e., a pricing policy that specifies a quantity-price pair). The optimal all-units discount and the optimal incremental discount can be obtained by solving a similar problem. The authors were able to show that, both analytically and numerically, the fixed-package pricing policy dominates the optimal all-units discount, which in term outperforms the optimal incremental discount, when expected profit is concerned.

Altintas, Erhun and Tayur [3] addressed the problem of designing the optimal all-units quantity discount with a single price break from the perspective of a supplier that faces a newsvendor-type buyer in a multiple-period setting. It is assumed that, for every period in the planning horizon except the last one, leftover units at the end of one period will be carried over to the next period and unfilled demand will be backlogged at a cost. At the end of the planning horizon, leftover units must be disposed and unfilled demand must be satisfied from an outside supply source. The supplier is concerned with maximizing its own profit, which is the amount of money coming from the retailer less the trucking cost. The trucking cost for each order of size $Q$ can be expressed as $K\left\lceil\frac{Q}{C}\right\rceil$, where $K$ is the fixed operating cost per truck and $C$ is the capacity of each truck. The buyer seeks to minimize her average cost. The authors first considered a single period model that allows positive initial inventory level $y$. Let $k_{i}$ denote the critical fractile value corresponding to the whole sale price $v_{i}, i=0,1$. Define $S_{i}=F^{-1}\left(k_{i}\right), i=0,1$, and $S_{01}$ as some value that equates the resulting expected costs of ordering $q_{1}$ or ordering up to $S_{0}$ if necessary. The authors demonstrated that a three-index policy with indices $\left(S_{0}, S_{1}, S_{01}\right)$ can be the buyer's optimal ordering policy. To be specific, order $\max \left\{S_{1}-y, q_{1}\right\}$ if the initial inventory level y is less than $S_{01}$ and order $\max \left\{S_{0}-y, 0\right\}$ otherwise. However, this three-index policy does not apply to a multi-period model according to the numerical study. One interesting finding is that demand variance and the variance of orders not including the orders of size 0 may move in opposite directions, since the buyer may follow polices with fewer indices under more variant demand. For the supplier's problem, fixing $v_{1}$ and treating $v_{0}$ and $q_{1}$ as variables, the authors were able to show numerically that the supplier should offer quantity discounts that can act as minimum order quantity schemes to reduce the order variance when the fixed cost $K$ is high.

\footnotetext{
${ }^{10}$ Stochastic dominance implies that the cumulative density function of demand for buyer $i$ is strictly greater than the cumulative density function for buyer $j$ for all possible values of $x([38])$.
} 
Lau, Lau and Wang [33] investigated the design of a supplier's wholesale pricing policy in the form of an all-units discount with a single price-break point. They considered a supply chain with a single Stackelberg-leader supplier and numerous heterogeneous newsvendor-type retailers. Each retailer $i$ faces a normally distributed demand with mean $\mu_{i}$ and standard deviation $\sigma_{i}$, where $\sigma_{i}=k \mu_{i}$ (where $k$ is a common scale parameter across all retailers) and all $\mu_{i}$ 's follow another stochastic distribution $H_{3}(\cdot)$ by assumption. According to the authors, all demand characteristics are to be estimated from empirical data and they assumed that the parameters $k, p$ (market price) and $c$ (retailer specific cost) are identical for all retailers. Based on a numerical analysis, they concluded that for a Stackelberg-leader supplier whose only objective is profit maximization, the optimal all-units quantity discount dominates the optimal single wholesale price scheme and that retailers' heterogeneity, indicated by the standard deviation of $H_{3}(\cdot)$, adversely affects the effectiveness of the quantity discount scheme. In addition, for a profit-maximizing supplier that is also concerned with allocating a certain amount of profits to the retailers, the optimal quantity discount again performs better than the optimal single wholesale price scheme in terms of the supplier's expected profit.

\section{Buyer Risk Profile}

This part of the paper is mainly devoted to the analysis of the ordering policies of newsvendors with various risk preferences, including, but not limited to, risk-averse and risk-seeking preferences. Motivated by empirical and experimental observations, alternative risk preferences (such as lossaversion, and stock-out aversion) have been analyzed in the context of the newsvendor setting. General utility functions, mean-variance, and coherent risk measures are the three most popular approaches to date. In what follows, we will introduce some general results for a risk-averse newsvendor derived and provide a detailed review of other contributions in this domain.

Eeckhoudt, Gollier and Schlesinger [20] provided a detailed investigation of the effects of risk, risk aversion and changes in various price and cost parameters for a risk-averse retailer. One important assumption that simplifies the analysis is that all demand must be satisfied. To be specific, when the initial order is insufficient to fulfill the demand, the retailer purchases additional products at a cost of $\hat{v}$, which is higher than the initial order cost $v$. The profit function can then be reformulated as

$$
\Pi(x, Q)= \begin{cases}\Pi_{-}(x, Q)=w_{0}+p x-v Q+g(Q-x), & x \leq Q \\ \Pi_{+}(x, Q)=w_{0}+p Q-v Q-\hat{v}(x-Q), & x>Q\end{cases}
$$

where $w_{0}$ is the newsvendor's initial wealth. Obviously, for a given $Q$, profit increases in demand $x$, 
which is defined in the interval $[0, T]$. For a risk-averse newsvendor, the objective is to maximize the expected utility of the random profit. Let $u(\cdot)$ denote the increasing and concave utility function. The problem can be stated as max $E[u(\Pi(x, Q))]$, where

$$
E[u(\Pi(x, Q))]=\int_{0}^{Q} u\left[\Pi_{-}(x, Q)\right] f(x) d x+\int_{Q}^{T} u\left[\Pi_{+}(x, Q)\right] f(x) d x
$$

The first and second derivatives of the objective function with respect to $Q$ are as follows.

$$
\begin{aligned}
\frac{\partial E[u(\Pi(x, Q))]}{\partial Q}= & (g-v) \int_{0}^{Q} u^{\prime}\left[\Pi_{-}(x, Q)\right] f(x) d x+(\hat{v}-v) \int_{Q}^{T} u^{\prime}\left[\Pi_{+}(x, Q)\right] f(x) d x \\
\frac{\partial^{2} E[u(\Pi(x, Q))]}{\partial^{2} Q}= & (g-v)^{2} \int_{0}^{Q} u^{\prime \prime}\left[\Pi_{-}(x, Q)\right] f(x) d x-(\hat{v}-g) u^{\prime}[p Q-v Q] f(Q) \\
& +(\hat{v}-v)^{2} \int_{Q}^{T} u^{\prime \prime}\left[\Pi_{+}(x, Q)\right] f(x) d x
\end{aligned}
$$

It is relatively easy to show that the objective function $E[u(\Pi(x, Q))]$ is concave in $Q$ and thus the optimal order quantity can be determined by first-order condition. Let $Q_{N}^{*}$ and $Q_{A}^{*}$ denote the risk-neutral and risk-averse optimal order quantities, respectively. Since

$$
\begin{array}{ll}
u^{\prime}\left[\Pi_{-}(x, Q)\right] \geq u^{\prime}[p Q-v Q], & x \leq Q \\
u^{\prime}\left[\Pi_{+}(x, Q)\right]<u^{\prime}[p Q-v Q], & x>Q
\end{array}
$$

we get:

$$
\begin{aligned}
\frac{\partial E\left[u\left(\Pi\left(x, Q_{A}^{*}\right)\right)\right]}{\partial Q}= & (g-v) \int_{0}^{Q_{N}^{*}} u^{\prime}\left[\Pi_{-}\left(x, Q_{N}^{*}\right)\right] f(x) d x \\
& +(\hat{v}-v) \int_{Q_{A}^{*}}^{T} u^{\prime}\left[\Pi_{+}\left(x, Q_{A}^{*}\right)\right] f(x) d x \\
< & u^{\prime}\left(p Q_{N}^{*}-v Q_{N}^{*}\right) \cdot\left[(g-v) \int_{0}^{Q_{A}^{*}} f(x) d x+(\hat{v}-v) \int_{Q_{N}^{*}}^{T} f(x) d x\right] \\
= & 0
\end{aligned}
$$

This indicates that a risk-averse newsvendor orders less than a risk-neutral newsvendor in this setting. Keren and Pliskin [29] also showed that, under uniformly distributed demand, the optimal order quantity decreases in the degree of risk aversion even if penalty is incurred for lost sales. As an increase in risk aversion can be modeled by a concave transformation of the current utility function, it is not so difficult to show that $Q_{A}^{*}$ decreases in the degree of risk aversion. Eeckhoudt, Gollier and Schlesinger [20] showed that increases in the salvage value and the re-order cost both leads to a larger initial order, while the effect of changes in the initial order cost $v$ on $Q_{A}^{*}$ is still 
ambiguous. As for the changes in retail price, the authors showed that $Q_{A}^{*}$ increases in $p$ if the retailer's preference displays decreasing partial relative risk aversion. The effects of increases in two types of risk were examined in this paper, risk associated with the initial wealth and demand risk. It is shown that the optimal size of the initial order is unaffected by the additional risk if the utility is quadratic or exhibits constant absolute risk aversion, decreases if the utility displays decreasing absolute risk aversion. Riskier demand is described by a probability distribution $G$ which is a mean-preserving with an increase in risk (MIR) of $F$. Let $Q_{A F}^{*}$ and $Q_{A G}^{*}$ denote the optimal order quantity associated with demand distribution $F$ and $G$, respectively. The authors were able to prove that (1) $Q_{A F}^{*}>Q_{A G}^{*}$ if an MIR is restricted to $\left[0, Q_{A F}^{*}\right]$ or $G$ and $F$ satisfies the inequality $[G(x)-F(x)][Q-x] \geq 0,(2) Q_{A F}^{*}<Q_{A G}^{*}$ if MIR is restricted to $\left[Q_{A F}^{*}, T\right]$.

Lau [34] provided solution procedures for solving the newsvendor problems under two alternative optimization objectives, maximizing expected utility and maximizing the probability of achieving a target profit level. Let $A$ denote the actual sales of the newsvendor. The profit can be expressed as $\Pi(x, Q)=(p-g+B) A-B x-(v-g) Q$, where $A=\min (x, Q)$. When only the tradeoff between mean and standard deviation of the profit is considered, the optimization problem becomes $\max E[\Pi(x, Q)]-k \sqrt{\operatorname{var}(\Pi(x, Q))}$, where $k$ is some constant that reflects the decision maker's degree of risk aversion. The author demonstrated that the optimal solution to the classic newsvendor problem always serves as an upper bound on the optimal order quantity in this scenario. Besides, he suggested determining the optimal solution value numerically due to the complexity of the first-order condition. For the case in which the decision maker is concerned with maximizing the profit's expected utility, the author considered a utility function approximated by an $\mathrm{n}^{\text {th }}$ degree polynomial, $u(\Pi(x, Q))=\sum_{i=0}^{n} a_{i} \Pi^{i}(x, Q)$ and derived the corresponding first-order condition. Under the objective of maximizing the probability of achieving a target profit level $T$, i.e., $\max \operatorname{prob}(\Pi(x, Q) \geq T)$, the optimal order quantity is equal to $T /(p-v)$ when $B=0$, which is independent of demand distribution. When $B>0$, the first order condition can be expressed as $\frac{f\left(D_{1}\right)}{f\left(D_{2}\right)}=\frac{(p-v+B)(p-g)}{B(v-g)}$, where $D_{1}$ and $D_{2}$ are the two demand realizations that generates the target profit level T. However, no conclusion can be reached regarding the behavior of the objective function for an arbitrary demand distribution.

Bouakiz and Sobel [10] showed the optimality of a base-stock policy for a multi-period newsvendor problem in which the newsvendor's risk attitude can be described by an exponential utility function. Specifically, they assumed a utility function $u(C(N))=-e^{-\tau C(N)}$, where $\tau$ is some positive constant measuring the risk-aversion degree of the retailer and $C(N)$ is the present value of the total cost incurred in the N-period planning horizon. Let $r$ denote the discount factor. With the infinite-horizon model, the authors proved the existence of a functions $y(\cdot)$ that gives the optimal 
base stock level for period $i$ in the form of $y\left(r^{i-1} \tau\right)$, which eventually approaches the optimal base stock level for a risk-neutral retailer.

Agrawal and Seshadri [1] considered a similar problem as Eeckhoudt, Gollier and Schlesinger [20] except that the demand now depends on the retail price. Assuming that the mean demand is equal to $a(p)$, which is a decreasing and concave function of $p$, the authors modeled the effect of price on demand in two ways: (1) $x=a(p) \epsilon_{1}$, where $\epsilon_{1}$ is a random variable with unity mean; (2) $x=a(p)+\epsilon_{2}$, where $\epsilon_{2}$ is a random variable with zero mean. The authors first reduce the bivariate objective function to a univariate function through a graphical analysis of price $p$ versus all possible demand realizations, and then examine the impact of risk aversion on the retailer's pricing and ordering decisions. With the multiplicative demand model (model 1), it is demonstrated that the optimal retail price increases in the degree of risk aversion, while the optimal size of the initial order decreases. With the additive demand model (model 2), a more risk-averse retailer will choose a lower retailer price, but the effect of risk aversion on the optimal initial order quantity cannot be precisely identified.

Schweitzer and Cachon [42] provided a complete investigation of the relationship between the newsvendor's profit-maximizing order quantity and optimal order quantities under various alternative objectives. A symmetric demand distribution and zero shortage cost are assumed in the analysis. Let $w_{0}$ denote the initial wealth. For a loss-averse newsvendor, the authors considered a utility function in which $u(w)$ is equal to $w$ for $w \geq w_{0}$ and $\lambda w$ otherwise, where $\lambda$ is a measure of the newsvendor's degree of loss aversion. It is proved that the optimal order quantity of a loss-averse newsvendor is smaller than the profit-maximizing order quantity and decreases in $\lambda$. For a newsvendor with preference for waste-aversion (dislike of excess inventory), stock-out aversion, or minimizing ex-post inventory error (minimizing absolute differences between $Q$ and $x$ ), the authors modeled the respective utilities as expected profit less the cost incurred by the corresponding deviation. They showed that a waste-averse newsvendor orders less than a risk-neutral newsvendor, while a stockout-averse newsvendor orders more. By defining products with the critical fractile greater than $1 / 2$ as high-profit products and as low-profit products otherwise, then to minimize ex-post inventory error, the newsvendor tends to order less than profit-maximizing quantity for high-profit products and more for low-profit products. The same ordering policy applies to newsvendors adopting an anchoring policy in which the decision maker anchors on mean demand and adjusts the profit-maximizing order quantity. The results derived from the last two situations are exactly what the authors observed in their experiments.

Arcelus, Kumar and Srinivasan [6] investigated the pricing, ordering and promotion policies of a risk-sensitive (risk-averse or risk-seeking) newsvendor under price-dependent and stochastic 
demand. Three promotion schemes are considered in this paper: rebate, advertising and the simultaneous use of the previous two schemes. It is assumed that the price and promotion decisions (i.e., rebate's face value and advertising expenditure) only affect the deterministic part of demand. To be specific, price and rebate decisions have an exponential relationship with demand, while advertising enters in logarithmic form. Both additive and multiplicative demand errors are allowed in this model. It is also worth mentioning that in contrast to most of the previous papers we have reviewed, risk preferences are included in this model by introducing a risk-adjustment factor $\lambda$ for the stochastic part of the expected profit. $\lambda>1$ for risk-averse newsvendors and $\lambda<1$ for riskseeking decision makers. Necessary optimality conditions are derived for all the possible decisions to be made for both additive error and multiplicative error. It is demonstrated that both the optimal order quantity and the optimal price are independent of the advertising expenditure. Since no closed form solution can be achieved due to technical complexity, numerical analysis is conducted for an example with uniformly distributed demand. One observation is that if no promotion scheme is available, price and expected profit increases in the value of $\lambda$, but order quantity falls.

Many researchers have considered the use of financial pricing and risk measures in inventory problems. Anvari [4] argued that the mean-variance approach proposed in Lau [34] may not apply to a decision maker facing with multiple investment options since the effect of covariance cannot be captured by the mean-variance approach. The author hence suggested the use of the capital asset pricing model (CAPM) in the inventory models and thus optimizing from the perspective of the shareholders, instead of the managers, to avoid agency problem. Consider a newsvendor with two investment options, the inventory investment option (purchase $Q$ units at a price of $v$ ) and the option representing all the other investment opportunities other than the inventory option with a random return rate. It is assumed that $B=0$ and that investment is made at the beginning of the single period while returns will be collected at the end of the period. Let $C$ denote the total capital available for investment. The objective is now to determine $Q$ maximizing the shareholder's wealth represented by $[E[D(Q)]-\Omega \operatorname{Cov}(D(Q), M)] /\left(1+r_{f}\right)-C$, where $D(Q)$ is the random liquidating dividend, $\Omega$ is the market price per unit of risk, $M$ is the value of the market portfolio at the end of period and $r_{f}$ is the risk-free rate. The behavior of $\operatorname{Cov}(D(Q), M)$ was examined in this paper under the assumption that the joint distribution of demand $x$ and $M$ is bivariate normal. (A solution procedure was developed in Chung [18] for this special case. ) Moreover, the author showed that the proposed model can be reduced to the classic newsvendor model by: (1) setting $r_{f}=0 ;(2)$ dropping out the term $\operatorname{Cov}(D(Q), M)$ since risk is not considered in the classic setting; and (3) ignoring the return from the other investment option.

Gaur and Seshadri [21] investigated the problem of hedging operational risk using financial 
instruments in the newsvendor environment. Demand is assumed to be correlated with the price of a financial asset in a complete financial market with a unique risk-neutral pricing measure. No penalty is considered for lost sales. The decision maker makes the inventory decision at time 0 and sells the products at time $T$. Let $S_{T}$ denote the price of the financial asset at time $T$. Demand is modeled as $x=a+b S_{T}$ when demand is perfectly correlated with the asset price and as $x=a+b S_{T}+\epsilon$ when demand is only partially correlated. $\epsilon$ is the decision maker's subjective forecast error. The authors were able to show that, for a risk-neutral decision maker, the optimal order quantity is not affected by the hedging decision in both cases. However, hedging transactions enable greater reduction in risk when demand is more correlated with the price of the asset. Under certain conditions on the structure of the hedging portfolio and the decision maker's utility function, financial hedging increases the optimal order quantity if a risk-averse decision maker is willing to invest in inventory rather than put all the money in the financial market.

Ahmed, Cakmak and Shapiro [2] suggested the use of coherent risk measures in inventory problems to obtain convex objective function. They analyzed a multi-period inventory problem with linear costs. The total cost for the single-period problem is expressed as $C(Q)=v Q-\gamma g(Q-$ $x)+B[x-Q]^{+}+h[Q-x]^{+}$, where $\gamma$ is a discount parameter within the interval $(0,1]$ and $h$ is the holding cost for each unit left. Let $\rho(\cdot)$ denote a coherent risk measure. When risk aversion is considered, the problem can be formulated as min $\rho(C(Q))$. For an arbitrary coherent risk measure, the authors proved its one-to-one correspondence with the min-max formulation. Using a mean-absolute deviation risk measure in the form of $\rho(z)=E[z]+\lambda E[|z-E[z]|], \lambda \in[0,1 / 2]$, the structure of the optimal solution is found to be similar to that of the risk-neutral case for the multi-period problem.

Gotoh and Takano [23] investigated the risk-sensitive newsvendor problem under the objective of minimizing the conditional value-at-risk $(\mathrm{CVaR})$, which is a coherent risk measure. Let $\Delta(x, Q)$ denote a particular loss function of interest and $\alpha$ denote some pre-specified target level of the loss function. The CVaR minimization problem formulated in this paper is to find the minimizing $Q$ and $\alpha$ values of the function $\alpha+\frac{1}{1-\beta} E\left[[\Delta(x, Q)-\alpha]^{+}\right]$for a given $\beta$. The objective function is introduced in Rockafellar and Uryasev [41]. The parameter $\beta$ refers to the probability of the loss function exceeding the infimum of all the $\alpha$ values that satisfy the inequality $\Phi(\alpha \mid Q) \geq \beta$, where $\Phi(\cdot)$ is defined as the distribution function of $\Delta(x, Q)$. Two loss functions are considered in this paper, the net loss $-\Pi(Q)$ and the total cost $C(Q)$. The authors proved the convexity of the objective function and derived closed form expressions for $Q$ and $\alpha$ in both cases. The two CVaR minimization problems are shown to be generalizations of the classic newsvendor problem. However, unlike what happens in the classic newsvendor case where maximizing profit is equivalent 
to minimizing cost, the two sets of $\left(Q^{*}, \alpha^{*}\right)$ can be very different from each other. Analytical results are also obtained with the mean-risk criterion where risk is measured by the net loss CVaR. A linear programming formulation is also developed for scenarios where the decision maker has multiple products to handle.

Jammernegg and Kischka [25] analyzed the ordering policy and its corresponding performance measures, such as cycle service level (CSL)and probability of loss, of a risk-sensitive newsvendor under the mean-deviation criterion. No penalty cost is considered. The problem is formulated as $\max _{(Q, \alpha)} \frac{\lambda-\alpha}{1-\alpha} C \operatorname{VaR}_{\alpha}(Q)+\frac{1-\lambda}{1-\alpha} E[\Pi(Q)]$, where $C V a R_{\alpha}(Q)=E\left[\Pi(Q) \mid \Pi(Q) \leq F^{-1}(\alpha)\right], 0<\alpha \leq 1$, $0 \leq \lambda \leq 1$. This objective applies to a risk-neutral newsvendor if $\alpha=\lambda$, a risk-averse newsvendor if $\alpha<\lambda$, and to a risk-seeking newsvendor if $\alpha>\lambda$. The closed form expression for the optimal order quantity is also derived under this criterion. Specifically, $Q^{*}=F^{-1}\left(\frac{p-v}{p-g}+\frac{\alpha-\lambda}{1-\lambda} \cdot \frac{v-g}{p-g}\right)$ for $\lambda \leq \frac{p-v}{p-g}$, and $F^{-1}\left(\frac{p-v}{p-g} \cdot \frac{\alpha}{\lambda}\right)$, otherwise. The conclusion is reached that a risk-averse (risk-seeking) newsvendor orders less (more) than a risk-neutral newsvendor does. The cycle service level, probability of loss, defined as $P\left(\Pi\left(Q^{*}\right) \leq 0\right)$, and expected loss, defined as $E\left[\Pi\left(Q^{*}\right) \mid \Pi\left(Q^{*}\right) \leq 0\right]$, increase in $\alpha$ and decrease in $\lambda$. In addition to the conditional expected value approach discussed above, the authors also suggested using performance measures to model the decision maker's risk preference. For example, a decision maker is risk averse (risk-seeing) if his/her self-specified CSL is less (greater) than the optimal CSL in the classic newsvendor scenario.

Wang and Webster [47] examined the ordering policy of a loss-averse newsvendor. They used the same utility function to describe the loss-aversion preference as in Schweitzer and Cachon [42] but assumed a positive shortage cost. Let $q_{1}(Q)=(v-g) Q$ and $q_{2}(Q)=\frac{p-v+B}{B} Q$. The objective function can be written as $E[u(\Pi(Q, x))]=E[\Pi(Q, x)]+(\lambda-1)\left[\int_{0}^{q_{1}(Q)} \Pi_{-}(x, Q) d F(x)+\right.$

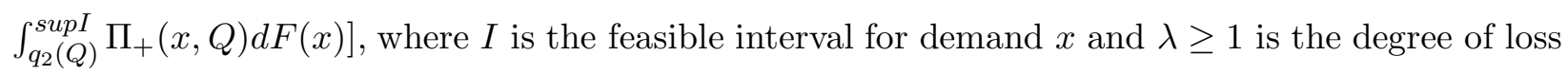
aversion. The two integrals in the objective function represent the expected underage and overage losses and have been explictly defined earlier in the discussion of Eeckhoudt, Gollier and Schlesinger [20]. Define $(p-v+B)\left(1-F\left(q_{2}(Q)\right)\right)$ as the marginal underage loss and $(v-g) F\left(q_{1}(Q)\right)$ as the marginal overage cost. The authors showed that, for $\lambda>1$, whether a loss-averse newsvendor orders more or less than a risk-neutral newsvendor depends on the relative magnitude of the marginal underage and overage losses, which in terms depends on the demand distribution and the values of the price and cost parameters. If the marginal underage loss exceeds the marginal overage loss, the loss-averse newsvendor tends to order more than a risk-neutral decision maker. However, when the shortage cost is lower than some threshold value, the loss-averse optimal order quantity is always less than the profit-maximizing order quantity and decreases in the degree of loss-aversion. And it is not surprising to know that the loss-averse optimal order quantity increases in the shortage cost. 


\section{Conclusions and Directions for Future Research}

In this paper, we have summarized the existing contributions on the newsvendor problem for managing inventories. Over and above synthesizing, summarizing, and extending the current contributions, we also find that there are several opportunities for future research. These are as follows:

- Supplier capacity constraints are generally not considered in the newsvendor setting. More recently, Burke, Carrillo, and Vakharia [11] have examined how a fixed capacity constraint would moderate the newsvendor's ordering decision. However, what has not been addressed is how the supplier could moderate the capacity decision (i.e., increase or decrease capacity) in this setting.

- One extension which has of late been receiving greater interest is that of integrating both stochastic demand and stochastic supply in newsvendor ordering decisions. A first attempt to address this issue is the research of Burke, Carrillo, and Vakharia [12] where the authors show that under assumptions of uniformly distributed demand, closed form solutions could be obtained regardless of the underlying distribution of supplier reliabilities. It would be interesting to examine whether their analysis holds for other demand distributions and also whether, it can be extended to multiple stochastic demand sources.

- The idea of zero supplier lead time of supply is another assumption in the newsvendor setting. Given that stochastic lead times are quite common in certain settings (e.g., sourcing of seasonal goods), how these lead times impact the buyer's ordering decision is another area for future research.

- There has been some prior research on the multi-period newsvendor's ordering decision (see [43] for a review of this problem). However, this has not addressed the dynamics of pricing decisions, marketing effort, and/or buyer penalties in changing ordering quantities which are obviously relevant in an applied setting.

In addition to the suggestions noted above, it is obvious that future research opportunities which integrate these distinct areas are also relevant for future research. For example, practical settings where capacity and supplier price are related would obviously moderate the ordering policy for a newsvendor. Further, it might also be worthwhile to investigate settings where buyers are charged a penalty for accepting quantities which are lower than those previously agreed upon since this would tend to increase supplier prices. 
Figure 1: Graphical Illustration of Alternative Pricing Schemes

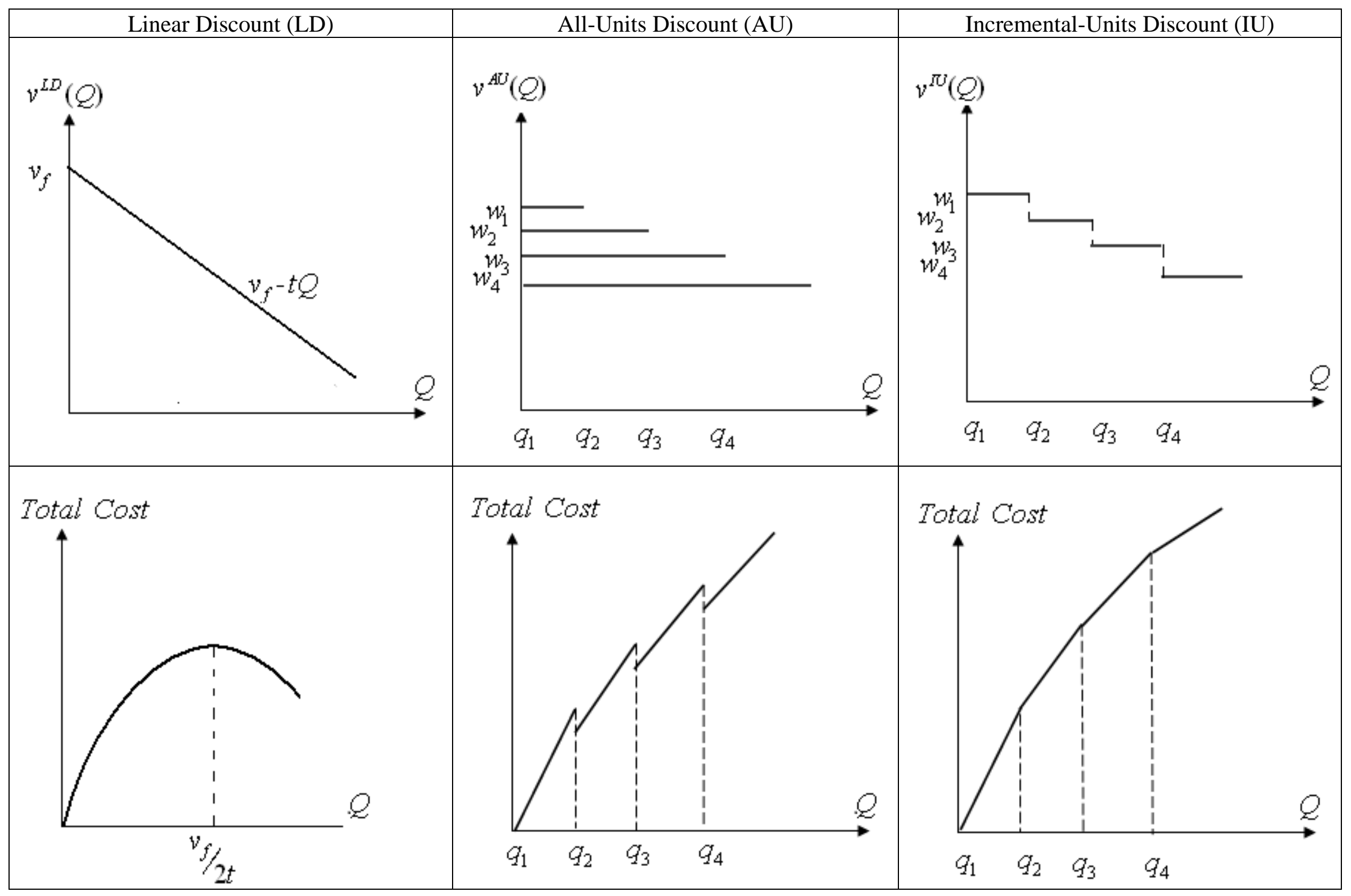


Figure 2: Algorithm for the All-Units discount scheme

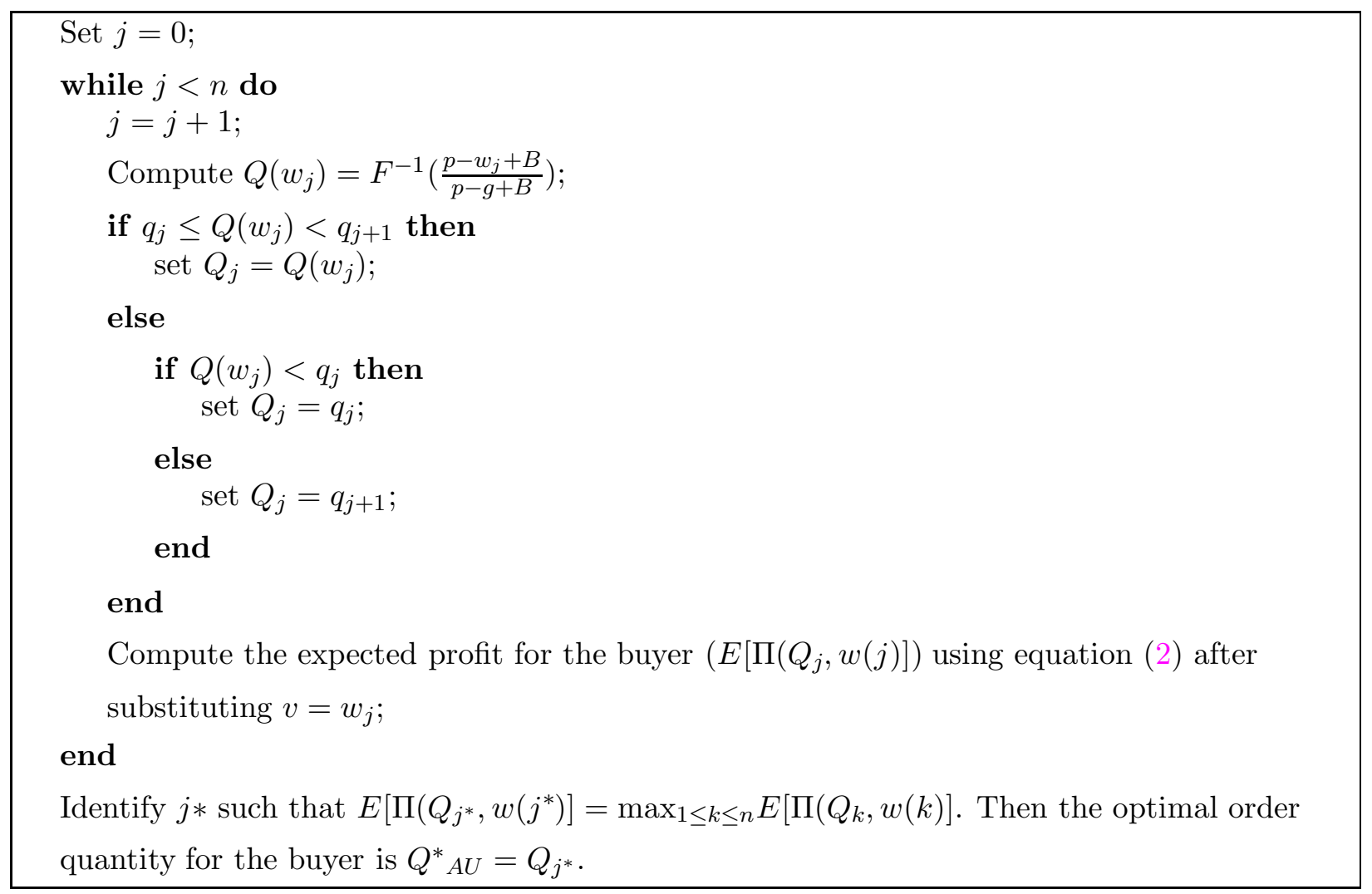




\section{Figure 3: Algorithm for the Incremental-Units discount scheme}

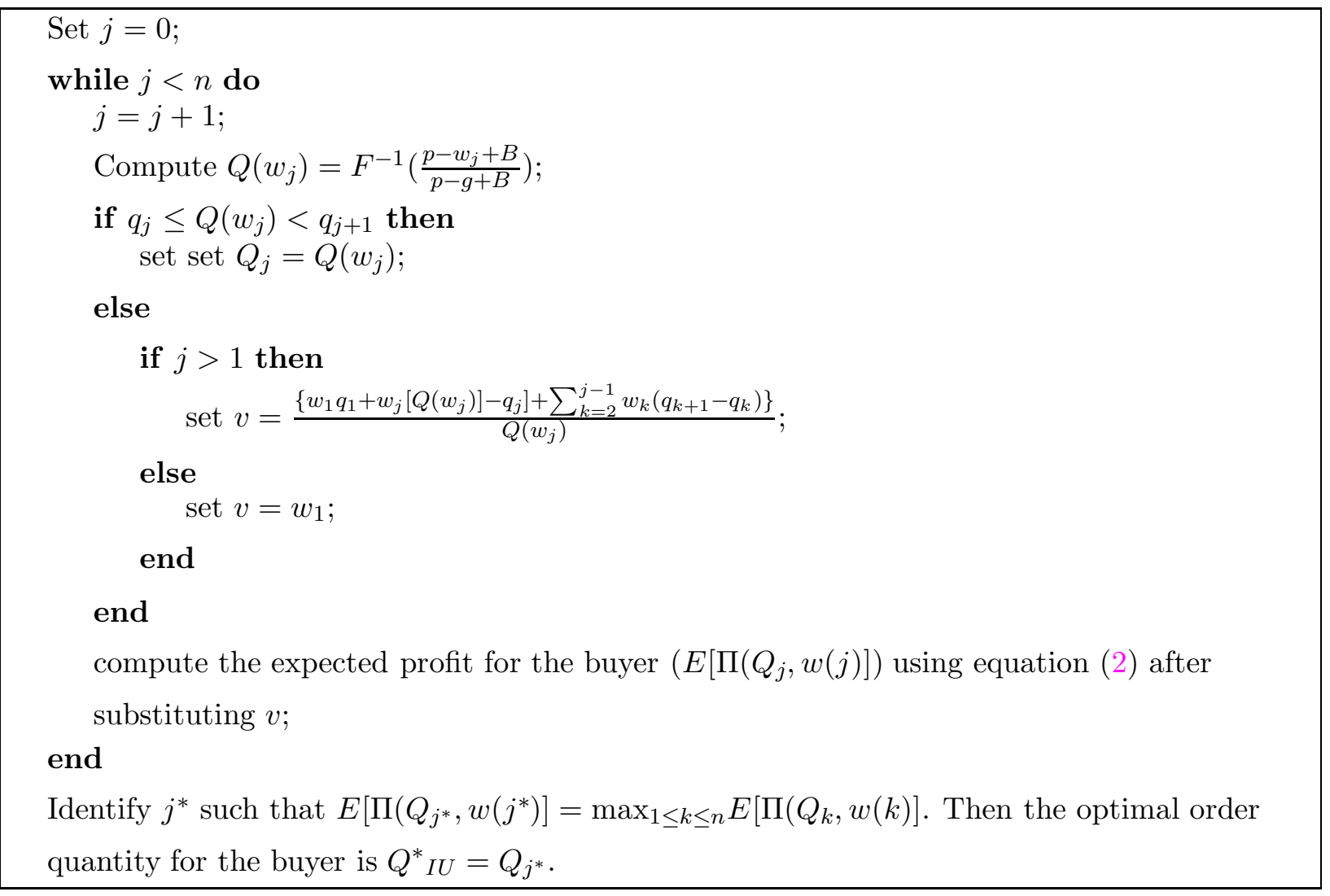




\section{References}

[1] V. Agrawal and S. Seshadri. Impact of uncertainty and risk aversion on price and order quantity in the newsvendor problem. Manufacturing $\&$ Service Operations Management, 2 (4):410-423, 2000.

[2] S. Ahmed, U. Cakmak, and A. Shapiro. Coherent risk measures in inventory problems. European Journal of Operational Research, 182(1):226-238, 2007.

[3] N. Altintas, F. Erhun, and S. Tayur. Quantity discounts under demand uncertainty. Management Science, 54(4):777-792, 2008.

[4] M. Anvari. Optimality criteria and risk in inventory models: The case of the newsboy problem. The Journal of the Operational Research Society, 38(7):625-632, 1987.

[5] F.J. Arcelus, S. Kumar, and G. Srinivasan. Retailer's response to alternate manufacturer's incentives under a single-period, price-dependent, stochastic-demand framework. Decision Sciences, 36 (4):599-626, 2005.

[6] F.J. Arcelus, S. Kumar, and G. Srinivasan. Pricing, rebate, advertising and ordering policies of a retailer facing price-dependent stochastic demand in newsvendor framework under different risk preferences. International Transactions on Operations Research, 13(3):209-227, 2006.

[7] K. Arrow, T. Harris, and J. Marshak. Optimal inventory policy. Econometrica, 19 (3):250-272, 1951.

[8] R.C. Baker and T.L. Urban. Single-period inventory dependent demand models. Omega Int. J. of Mgmt Scie., 16(6):605-607, 1988.

[9] A. Balakrishnan, M.S. Pangburn, and E. Stavrulaki. Integrating the promotional and service roles of retail inventories. Manufacturing $\&$ Service Operations Management, 10(2):218-235, 2008.

[10] M. Bouakiz and M.J. Sobel. Inventory control with an exponential utility criterion. Operations Research, 40(3):603-608, 1992.

[11] G.J. Burke, J.E. Carrillo, and A.J. Vakharia. Single versus multiple supplier sourcing strategies. European Journal of Operational Research, 182 (1):95-112, 2007.

[12] G.J. Burke, J.E. Carrillo, and A.J. Vakharia. Sourcing decisions with stochastic supplier reliability and stochastic demand. Production $\&$ Operations Management, 18(4):475-484, 2009. 
[13] G.J. Burke and A.J. Vakharia. Supply Chain Management, The Internet Encyclopedia. Wiley, New York, 2004.

[14] A. Burnetas, S.M. Gilbert, and C.E. Smith. Quantity discounts in single-period supply contracts with asymmetric demand information. IIE Transactions, 39(5):465-479, 2007.

[15] G.P. Cachon. Supply chain coordination with contracts. In T. de Kok S. Graves, editor, Handbooks in Operations and Management Science: Supply Chain Optimization. North-Holland Publishers, Amsterdam, The Netherlands, 2003.

[16] G.P. Cachon and M.A. Lariviere. Supply chain coordination with revenue-sharing contracts: Strengths and limitations. Management Science, 51(1):30-44, 2005.

[17] F.Y. Chen, H. Yan, and L. Yao. A newsvendor pricing game. IEEE Transactions on Systems, Man, and Cybernetics, 34(4):450 - 456, 2004.

[18] K.H. Chung. Risk in inventory models: The case of the newboy problem - optimality conditions. The Journal of the Operational Research Society, 41(2):173-176, 1990.

[19] J.D. Dana and N.C. Petruzzi, Jr. Note: The newsvendor model with endogenous demand. Management Science, 47(11):1488-1497, 2001.

[20] L. Eeckhoudt, C. Gollier, and H. Schlesinger. The risk-averse (and prudent) newsboy. Management Science, 41(5):786-794, 1995.

[21] V. Gaur and S. Seshadri. Hedging inventory risk through market instruments. Manufacturing $\&$ Service Operations Management, 7(2):103-120, 2005.

[22] Y. Gerchak and D. Mossman. On the effect of demand randomness on inventories and costs. Operations Research, 40(4):804-807, 1992.

[23] J.Y. Gotoh and Y.C. Takano. Newsvendor solutions via conditional value-at-risk minimization. European Journal of Operational Research, 179(1):80-96, 2007.

[24] C.A. Ingene and M.E. Parry. Coordination and manufacturer profit maximization: The multiple retailer channel. Journal of Retailing, 71(2):129-151, 1995.

[25] W. Jammernegg and P. Kischka. Risk-averse and risk-taking newsvendors: a conditional expected value approach. Review of Management Science, 1(1):93-110, 2007.

[26] A.P. Jeuland and S.M. Shugan. Managing channel profits. Marketing Science, 2(3):239-271, 1983. 
[27] J.V. Jucker and M.J. Rosenblatt. Single-period inventory models with demand uncertainty and quantity discount: Behavioral implications and a new solution procedure. Naval Research Logistics Quarterly, 32(4):537-550, 1985.

[28] S. Karlin and C.R. Carr. Prices and optimal inventory policy. In H. Scarf K. Arrow, S. Karlin, editor, Studies in Applied Probability and Management Science. Stanford University Press, Standford, CA., 1962.

[29] B. Keren and J.S. Pliskin. A benchmark solution for the risk-averse newsvendor problem. European Journal of Operational Research, 174(3):1643-1650, 2006.

[30] B. Khouja. The single period (news-vendor) problem: literature review and suggestions for future research. Omega, 27:537-553, 1999.

[31] S. Kraiselburd, V.G. Narayanan, and A. Raman. Contracting in a supply chain with stochastic demand and substitute products. Production \& Operations Management, 13 (1):46-62, 2004.

[32] A.H.L. Lau and H.S. Lau. The newsboy problem with price-dependent demand distribution. IIE Transactions, 20(2):168-175, 1988.

[33] A.H.L. Lau, H.S. Lau, and J.C. Wang. Designing a quantity discount scheme for a newsvendortype product with numerous heterogeneous retailers. European Journal of Operational Research, 180(2):585-600, 2007.

[34] H.S. Lau. The newsboy problem under alternative optimization objectives. The Journal of the Operational Research Society, 31(6):525-535, 1980.

[35] C.S. Lin and D.E. Kroll. The single-item newsboy problem with dual performance measures and quantity discounts. European Journal of Operational Research, 100(3):562-565, 1997.

[36] E.S. Mills. Uncertainty and price theory. The Quarterly Journal of Economics, 73(1):116-130, 1959.

[37] P. Pantumsinchai and T.W. Knowles. Standard container size discounts and the single-period inventory problem. Decision Sciences, 22(3):612-619, 1991.

[38] J.E. Pecaric, Proschan F., and Tong Y.L. Convex Functions, Partial Orderings, and Statistical Applications. Academic Press, Boston,MA, 1992.

[39] N.C. Petruzzi and M. Dada. Pricing and the newsvendor problem: A review with extensions. Operations Research, 47(2):183-194, 1999. 
[40] L.H. Polatoḡlu. Optimal order quantity and pricing decisions in single-period inventory systems. International Journal of Production Economics, 23(1-3):175-185, 1991.

[41] R.T. Rockafellar and S. Uryasev. Conditional value-at-risk for general loss distributions. Journal of Banking and Finance, 26(7):14431471, 2002.

[42] M.E. Schweitzer and G.P. Cachon. Decision bias in the newsvendor problem with a known demand distribution: Experimental evidence. Management Science, 46(3):404-420, 2000.

[43] E.A. Silver, D.F. Pyke, and R. Peterson. Inventory Management and Production Planning and Scheduling. Wiley, NY, 1998.

[44] T.L. Urban. The interdependence of inventory management and retail shelf management. International Journal of Physical Distribution \& Logistics Management, 32(1):41-58, 2002.

[45] T.L. Urban. Inventory models with inventory-level-dependent demand: A comprehensive review and unifying theory. European Journal of Operational Research, 162(3):792-804, 2005.

[46] T.L. Urban and R.C. Baker. Optimal ordering and pricing policies in a single-period environment with multivariate demand and markdowns. European Journal of Operational Research, 103(3):573-583, 1997.

[47] C.X. Wang and S. Webster. The loss-averse newsvendor problem. Omega, 37(1):93-105, 2009.

[48] Z.K. Weng. Pricing and ordering strategies in manufacturing and distribution alliances. IIE Transactions, 29(8):681-692, 1997.

[49] Z.K. Weng. Coordinating order quantities between the manufacturing and the buyer: A generalized newsvendor model. European Journal of Operational Research, 156(1):148-161, 2004.

[50] T.M. Whitin. Inventory control and price theory. Management Science, 2(1):61-68, 1955.

[51] R.L. Zhan and Z.J.M. Shen. Newsvendor problem with pricing: Properties, algorithms, and simulation. In Proceedings of the 2005 Winter Simulation Conference, M.E., Kuhl, N.M., Steiger, F.B., Armstrong, J.A., Joines, eds., pages 1743-1748, 2005. 